\title{
A FAMILY OF DENSITY EXPANSIONS FOR LÉVY-TYPE PROCESSES
}

\author{
By Matthew Lorig ${ }^{1}$, Stefano Pagliarani And Andrea Pascucci \\ Princeton University, Università di Padova and Università di Bologna \\ To the memory of our dear friend and esteemed colleague Peter Laurence
}

\begin{abstract}
We consider a defaultable asset whose risk-neutral pricing dynamics are described by an exponential Lévy-type martingale subject to default. This class of models allows for local volatility, local default intensity and a locally dependent Lévy measure. Generalizing and extending the novel adjoint expansion technique of Pagliarani, Pascucci and Riga [SIAM J. Financial Math. 4 (2013) 265-296], we derive a family of asymptotic expansions for the transition density of the underlying as well as for European-style option prices and defaultable bond prices. For the density expansion, we also provide error bounds for the truncated asymptotic series. Our method is numerically efficient; approximate transition densities and European option prices are computed via Fourier transforms; approximate bond prices are computed as finite series. Additionally, as in Pagliarani, Pascucci and Riga (2013), for models with Gaussian-type jumps, approximate option prices can be computed in closed form. Sample Mathematica code is provided.
\end{abstract}

1. Introduction and literature review. A local volatility model is a model in which the volatility $\sigma_{t}$ of an asset $X$ is a function of time $t$ and the present level of $X$. That is, $\sigma_{t}=\sigma\left(t, X_{t}\right)$. Among local volatility models, perhaps the most well known is the constant elasticity of variance (CEV) model of Cox (1975). One advantage of local volatility models is that transition densities of the underlyingas well as European option prices-are often available in closed-form as infinite series of special functions [see Linetsky (2007) and references therein]. Another advantage of local volatility models is that, for models whose transition density is not available in closed form, accurate density and option price approximations are readily available [see Pagliarani and Pascucci (2012), e.g.]. Finally, Dupire (1994) shows that one can always find a local volatility function $\sigma(t, x)$ that fits the market's implied volatility surface exactly. Thus, local volatility models are quite flexible.

Despite the above advantages, local volatility models do suffer some shortcomings. Most notably, local volatility models do not allow for the underlying

Received April 2013; revised October 2013.

${ }^{1}$ Supported in part by NSF Grant DMS-07-39195.

MSC2010 subject classifications. 35R09, 60G99, 91G20, 91G80.

Key words and phrases. Local volatility, Lévy-type process, asymptotic expansion, pseudodifferential calculus, defaultable asset. 
to experience jumps, the need for which is well documented in literature [see Eraker (2004) and references therein]. Recently, there has been much interest in combining local volatility models and models with jumps. Andersen and Andreasen (2000), for example, discuss extensions of the implied diffusion approach of Dupire (1994) to asset processes with Poisson jumps (i.e., jumps with finite activity). And Benhamou, Gobet and Miri (2009) derive analytically tractable option pricing approximations for models that include local volatility and a Poisson jump process. Their approach relies on asymptotic expansions around small diffusion and small jump frequency/size limits. More recently, Pagliarani, Pascucci and Riga (2013) consider general local volatility models with independent Lévy jumps (possibly infinite activity). Unlike, Benhamou, Gobet and Miri (2009), Pagliarani, Pascucci and Riga (2013) make no small jump intensity/size assumption. Rather the authors construct an approximated solution by expanding the local volatility function as a power series. While all of the methods described in this paragraph allow for local volatility and independent jumps, none of these methods allow for state-dependent jumps.

Stochastic jump-intensity was recently identified as an important feature of equity models [see Christoffersen, Jacobs and Ornthanalai (2009)]. A locally dependent Lévy measure allows for this possibility. Recently, two different approaches have been taken to modeling assets with locally-dependent jump measures. Mendoza-Arriaga, Carr and Linetsky (2010) time-change a local volatility model with a Lévy subordinator. In addition to admitting exact option-pricing formulas, the subordination technique results in a locally-dependent Lévy measure. Jacquier and Lorig (2013) considers another class of models that allow for state-dependent jumps. The author builds a Lévy-type processes with local volatility, local default intensity, and a local Lévy measure by considering state-dependent perturbations around a constant coefficient Lévy process. In addition to pricing formula, the author provides an exact expansion for the induced implied volatility surface.

In this paper, we consider scalar Lévy-type processes with regular coefficients, which naturally include all the models mentioned above. Generalizing and extending the methods of Pagliarani, Pascucci and Riga (2013), we derive a family of asymptotic expansions for the transition densities of these processes, as well as for European-style derivative prices and defaultable bond prices. The key contributions of this manuscript are as follows:

- We allow for a locally-dependent Lévy measure and local default intensity, whereas Pagliarani, Pascucci and Riga (2013) consider a locally independent Lévy measure and do not allow for the possibility of default. A state-dependent Lévy measure is an important feature because it allows for incorporating local dependence into infinite activity Lévy models that have no diffusion component, such as Variance-Gamma [Madan, Carr and Chang (1998)] and CGMY/Kobol [Boyarchenko and Levendorskiı̌ (2002), Carr et al. (2002)]. 
- Unlike Benhamou, Gobet and Miri (2009), we make no small diffusion or small jump size/intensity assumption. Our formulae are valid for any Lévy type process with smooth and bounded coefficients, independent of the relative size of the coefficients.

- Whereas Pagliarani, Pascucci and Riga (2013) expand the local volatility and drift functions as a Taylor series about an arbitrary point, that is, $f(x)=$ $\sum_{n} a_{n}(x-\bar{x})^{n}$, in order to achieve their approximation result, we expand the local volatility, drift, killing rate and Lévy measure in an arbitrary basis, that is, $f(x)=\sum_{n} c_{n} B_{n}(x)$. This is advantageous because the Taylor series typically converges only locally, whereas other choices of the basis functions $B_{n}$ may provide global convergence in suitable functional spaces.

- Using techniques from pseudo-differential calculus, we provide explicit formulae for the Fourier transform of every term in the transition density and option-pricing expansions. In the case of state dependent Gaussian jumps, the respective inverse Fourier transforms can be explicitly computed, thus providing closed form approximations for densities and prices. In the general case, the density and pricing approximations can be computed quickly and easily as inverse Fourier transforms. Additionally, when considering defaultable bonds, approximate prices are computed as a finite sum; no numerical integration is required even in the general case.

- For models with Gaussian-type jumps, we provide pointwise error estimates for transition densities. Thus, we extend the previous results of Pagliarani, Pascucci and Riga (2013) where only the purely diffusive case is considered. Additionally, our error estimates allow for jumps with locally dependent mean, variance and intensity. Thus, for models with Gaussian-type jumps, our results also extend the results of Benhamou, Gobet and Miri (2009), where only the case of a constant Lévy measure is considered.

The rest of this paper proceeds as follows. In Section 2, we introduce a general class of exponential Lévy-type models with locally-dependent volatility, default intensity and Lévy measure. We also describe our modeling assumptions. Next, in Section 3, we introduce the European option-pricing problem and derive a partial integro-differential equation (PIDE) for the price of an option. In Section 4, we derive a formal asymptotic expansion (in fact, a family of asymptotic expansions) for the function that solves the option pricing PIDE (Theorem 1). Next, in Section 5, we provide rigorous error estimates for our asymptotic expansion for models with Gaussian-type jumps (Theorem 2). Lastly, in Section 6, we provide numerical examples that illustrate the effectiveness and versatility of our methods. Technical proofs are provided in the Appendix. Some concluding remarks are given in Section 7.

We mention specifically that the arguments needed to provide rigorous error estimates for our asymptotic expansions are quite extensive. As such, in this manuscript, we provide only an outline of the proof of Theorem 2 . The full proof 
of Theorem 2, as well as further numerical examples, can be found in the companion paper of Lorig, Pagliarani and Pascucci (2013).

2. General Lévy-type exponential martingales. For simplicity, we assume a frictionless market, no arbitrage, zero interest rates and no dividends. Our results can easily be extended to include locally dependent interest rates and dividends. We take, as given, an equivalent martingale measure $\mathbb{Q}$, chosen by the market on a complete filtered probability space $\left(\Omega, \mathcal{F},\left\{\mathcal{F}_{t}, t \geq 0\right\}, \mathbb{Q}\right)$ satisfying the usual hypothesis of completeness and right continuity. The filtration $\mathcal{F}_{t}$ represents the history of the market. All stochastic processes defined below live on this probability space and all expectations are taken with respect to $\mathbb{Q}$. We consider a defaultable asset $S$ whose risk-neutral dynamics are given by

$$
\begin{aligned}
S_{t} & =\mathbb{I}_{\{\zeta>t\}} e^{X_{t}} \\
d X_{t} & =\mu\left(t, X_{t}\right) d t+\sigma\left(t, X_{t}\right) d W_{t}+\int_{\mathbb{R}} d \bar{N}_{t}\left(t, X_{t-}, d z\right) z, \\
d \bar{N}_{t}\left(t, X_{t-}, d z\right) & =d N_{t}\left(t, X_{t-}, d z\right)-v\left(t, X_{t-}, d z\right) d t \\
\zeta & =\inf \left\{t \geq 0: \int_{0}^{t} \gamma\left(s, X_{s}\right) d s \geq \varepsilon\right\}
\end{aligned}
$$

Here, $X$ is a Lévy-type process with local drift function $\mu(t, x)$, local volatility function $\sigma(t, x) \geq 0$ and state-dependent Lévy measure $v(t, x, d z)$. We shall denote by $\mathcal{F}_{t}^{X}$ the filtration generated by $X$. The random variable $\mathcal{E} \sim \operatorname{Exp}(1)$ has an exponential distribution and is independent of $X$. Note that $\zeta$, which represents the default time of $S$, is constructed here trough the so-called canonical construction [see Bielecki and Rutkowski (2002)], and is the first arrival time of a doubly stochastic Poisson process with local intensity function $\gamma(t, x) \geq 0$. This way of modeling default is also considered in a local volatility setting in Carr and Linetsky (2006), Linetsky (2006), and for exponential Lévy models in Capponi, Pagliarani and Vargiolu (2014).

We assume that the coefficients are measurable in $t$ and suitably smooth in $x$ to ensure the existence of a solution to (1) [see Oksendal and Sulem (2005), Theorem 1.19]. We also assume the following boundedness condition which is rather standard in the financial applications: there exists a Lévy measure

$$
\bar{v}(d z):=\sup _{(t, x) \in \mathbb{R}^{+} \times \mathbb{R}} v(t, x, d z)
$$

such that

$$
\begin{aligned}
\int_{\mathbb{R}} \bar{v}(d z) \min \left(1, z^{2}\right) & <\infty, \quad \int_{|z| \geq 1} \bar{v}(d z) e^{z}<\infty, \\
\int_{|z| \geq 1} \bar{v}(d z)|z|<\infty &
\end{aligned}
$$


Since $\zeta$ is not $\mathcal{F}_{t}^{X}$-measurable, we introduce the filtration $\mathcal{F}_{t}^{D}=\sigma(\{\zeta \leq s), s \leq$ $t\}$ in order to keep track of the event $\{\zeta \leq t\}$. The filtration of a market observer then is $\mathcal{F}_{t}=\mathcal{F}_{t}^{X} \vee \mathcal{F}_{t}^{D}$. In the absence of arbitrage, $S$ must be an $\mathcal{F}_{t}$-martingale. Thus, the drift $\mu(t, x)$ is fixed by $\sigma(t, x), v(t, x, d z)$ and $\gamma(t, x)$ in order to satisfy the martingale condition ${ }^{2}$

$$
\begin{aligned}
& \mu(t, x)=\gamma(t, x)-a(t, x)-\int_{\mathbb{R}} v(t, x, d z)\left(e^{z}-1-z\right), \\
& a(t, x):=\frac{1}{2} \sigma^{2}(t, x) .
\end{aligned}
$$

We remark that the existence of the density of $X$ is not strictly necessary in our analysis. Indeed, since our formulae are carried out in Fourier space, we provide approximations of the characteristic function of $X$ and all of our computations are still formally correct even when dealing with distributions that are not absolutely continuous with respect to the Lebesgue measure.

3. Option pricing. We consider a European derivative expiring at time $T$ with payoff $H\left(S_{T}\right)$ and we denote by $V$ its no-arbitrage price. For convenience, we introduce

$$
h(x):=H\left(e^{x}\right) \quad \text { and } \quad K:=H(0)
$$

\section{PROPOSITION 1. The price $V_{t}$ is given by}

$$
V_{t}=K+\mathbb{I}_{\{\zeta>t\}} \mathbb{E}\left[e^{-\int_{t}^{T} \gamma\left(s, X_{s}\right) d s}\left(h\left(X_{T}\right)-K\right) \mid X_{t}\right], \quad t \leq T .
$$

The proof can be found in Section 2.2 of Linetsky (2006). Because our notation differs from that of Linetsky (2006), and because a short proof is possible by using the results of Jeanblanc, Yor and Chesney (2009), for the reader's convenience, we provide a derivation of Proposition 1 here.

ProOF OF Proposition 1. Using risk-neutral pricing, the value $V_{t}$ of the derivative at time $t$ is given by the conditional expectation of the option payoff

$$
\begin{aligned}
V_{t} & =\mathbb{E}\left[H\left(S_{T}\right) \mid \mathcal{F}_{t}\right] \\
& =\mathbb{E}\left[h\left(X_{T}\right) \mathbb{I}_{\{\zeta>T\}} \mid \mathcal{F}_{t}\right]+K \mathbb{E}\left[\mathbb{I}_{\{\zeta \leq T\}} \mid \mathcal{F}_{t}\right] \\
& =\mathbb{E}\left[h\left(X_{T}\right) \mathbb{I}_{\{\zeta>T\}} \mid \mathcal{F}_{t}\right]+K-K \mathbb{E}\left[\mathbb{I}_{\{\zeta>T\}} \mid \mathcal{F}_{t}\right] \\
& =K+\mathbb{I}_{\{\zeta>t\}} \mathbb{E}\left[e^{-\int_{t}^{T} \gamma\left(s, X_{s}\right) d s}\left(h\left(X_{T}\right)-K\right) \mid \mathcal{F}_{t}^{X}\right] \\
& =K+\mathbb{I}_{\{\zeta>t\}} \mathbb{E}\left[e^{-\int_{t}^{T} \gamma\left(s, X_{s}\right) d s}\left(h\left(X_{T}\right)-K\right) \mid X_{t}\right],
\end{aligned}
$$

\footnotetext{
${ }^{2}$ We provide a derivation of the martingale condition in Section 3, Remark 1 below.
} 
where we have used Corollary 7.3.4.2 from Jeanblanc, Yor and Chesney (2009) to write

$$
\mathbb{E}\left[\left(h\left(X_{T}\right)-K\right) \mathbb{I}_{\{\zeta>T\}} \mid \mathcal{F}_{t}\right]=\mathbb{I}_{\{\zeta>t\}} \mathbb{E}\left[\left(h\left(X_{T}\right)-K\right) e^{-\int_{t}^{T} \gamma\left(s, X_{s}\right) d s} \mid \mathcal{F}_{t}^{X}\right] .
$$

REMARK 1. By Proposition 1 with $K=0$ and $h(x)=e^{x}$, we have that the martingale condition $S_{t}=\mathbb{E}\left[S_{T} \mid \mathcal{F}_{t}\right]$ is equivalent to

$$
\mathbb{I}_{\{\zeta>t\}} e^{X_{t}}=\mathbb{I}_{\{\zeta>t\}} \mathbb{E}\left[e^{-\int_{t}^{T} \gamma\left(s, X_{s}\right) d s+X_{T}} \mid \mathcal{F}_{t}\right] .
$$

Therefore, we see that $S$ is a martingale if and only if the process $\exp \left(-\int_{0}^{t} \gamma(s\right.$, $\left.X_{s}\right) d s+X_{t}$ ) is a martingale. The drift condition (3) follows by applying the Itô's formula to the process $\exp \left(-\int_{0}^{t} \gamma\left(s, X_{s}\right) d s+X_{t}\right)$ and setting the drift term to zero.

From (4), one sees that, in order to compute the price of an option, we must evaluate functions of the form ${ }^{3}$

$$
v(t, x):=\mathbb{E}\left[e^{-\int_{t}^{T} \gamma\left(s, X_{s}\right) d s} h\left(X_{T}\right) \mid X_{t}=x\right] .
$$

By a direct application of the Feynman-Kac representation theorem [see, e.g., Pascucci (2011), Theorem 14.50], the classical solution of the following Cauchy problem:

$$
\left(\partial_{t}+\mathcal{A}^{(t)}\right) v=0, \quad v(T, x)=h(x),
$$

when it exists, is equal to the function $v(t, x)$ in (5), where

$$
\begin{aligned}
\mathcal{A}^{(t)} f(x)= & \gamma(t, x)\left(\partial_{x} f(x)-f(x)\right)+a(t, x)\left(\partial_{x}^{2} f(x)-\partial_{x} f(x)\right) \\
& -\int_{\mathbb{R}} v(t, x, d z)\left(e^{z}-1-z\right) \partial_{x} f(x) \\
& +\int_{\mathbb{R}} v(t, x, d z)\left(f(x+z)-f(x)-z \partial_{x} f(x)\right),
\end{aligned}
$$

is the characteristic operator of the $\operatorname{SDE}(1)$. In order to shorten the notation, in the sequel we will suppress the explicit dependence on $t$ in $\mathcal{A}^{(t)}$ by referring to it just as $\mathcal{A}$.

Sufficient conditions for the existence and uniqueness of solutions of secondorder elliptic integro-differential equations are given in Theorem II.3.1 of Garroni and Menaldi (1992). We denote by $p(t, x ; T, y)$ the fundamental solution of the

\footnotetext{
${ }^{3}$ Note: we can accommodate stochastic interest rates and dividends of the form $r_{t}=r\left(t, X_{t}\right)$ and $q_{t}=q\left(t, X_{t}\right)$ by simply making the change: $\gamma(t, x) \rightarrow \gamma(t, x)+r(t, x)$ and $\mu(t, x) \rightarrow \mu\left(t, X_{t}\right)+$ $r\left(t, X_{t}\right)-q\left(t, X_{t}\right)$.
} 
operator $\left(\partial_{t}+\mathcal{A}\right)$, which is defined as the solution of (6) with $h=\delta_{y}$. Note that $p(t, x ; T, y)$ represents also the transition density of $\log S^{4}$

$$
p(t, x ; T, y) d y=\mathbb{Q}\left[\log S_{T} \in d y \mid \log S_{t}=x\right], \quad x, y \in \mathbb{R}, t<T .
$$

Note also that $p(t, x ; T, y)$ is not a probability density since (due to the possibility that $\left.S_{T}=0\right)$ we have

$$
\int_{\mathbb{R}} p(t, x ; T, y) d y \leq 1 .
$$

Given the existence of the fundamental solution of $\left(\partial_{t}+\mathcal{A}\right)$, we have that for any $h$ that is integrable with respect to the density $p(t, x ; T, \cdot)$, the Cauchy problem (6) has a classical solution that can be represented as

$$
v(t, x)=\int_{\mathbb{R}} h(y) p(t, x ; T, y) d y .
$$

REMARK 2. If $\mathcal{G}$ is the generator of a scalar Markov process and dom $(\mathcal{G})$ contains $\mathcal{S}(\mathbb{R})$, the Schwartz space of rapidly decaying functions on $\mathbb{R}$, then $\mathcal{G}$ must have the following form:

$$
\begin{aligned}
\mathcal{G} f(x)= & -\gamma(x) f(x)+\mu(x) \partial_{x} f(x)+a(x) \partial_{x}^{2} f(x) \\
& +\int_{\mathbb{R}} v(x, d z)\left(f(x+z)-f(x)-\mathbb{I}_{\{|z|<R\}} z \partial_{x} f(x)\right),
\end{aligned}
$$

where $\gamma \geq 0, a \geq 0, v$ is a Lévy measure for every $x$ and $R \in[0, \infty$ ] [see Hoh (1998), Proposition 2.10]. If one enforces on $\mathcal{G}$ the drift and integrability conditions (2) and (3), which are needed to ensure that $S$ is a martingale, and allow setting $R=\infty$, then the operators (7) and (8) coincide (in the time-homogeneous case). Thus, the class of models we consider in this paper encompasses all nonnegative scalar Markov martingales that satisfy the regularity and boundedness conditions of Section 2.

REMARK 3. In what follows, we shall systematically make use of the language of pseudo-differential calculus. More precisely, let us denote by

$$
\psi_{\xi}(x)=\psi_{x}(\xi)=\frac{1}{\sqrt{2 \pi}} e^{i \xi x}, \quad x, \xi \in \mathbb{R},
$$

the so-called oscillating exponential function. Then $\mathcal{A}$ can be characterized by its action on oscillating exponential functions. Indeed, we have

$$
\mathcal{A} \psi_{\xi}(x)=\phi(t, x, \xi) \psi_{\xi}(x),
$$

\footnotetext{
${ }^{4}$ Here with $\log S$ we denote the process $X_{t} \mathbb{I}_{\{\zeta>t\}}-\infty \mathbb{I}_{\{\zeta \leq t\}}$.
} 
where

$$
\begin{aligned}
\phi(t, x, \xi)= & \gamma(t, x)(i \xi-1)+a(t, x)\left(-\xi^{2}-i \xi\right) \\
& -\int_{\mathbb{R}} v(t, x, d z)\left(e^{z}-1-z\right) i \xi+\int_{\mathbb{R}} v(t, x, d z)\left(e^{i \xi z}-1-i \xi z\right),
\end{aligned}
$$

is called the symbol of $\mathcal{A}$. Noting that

$$
e^{z \partial_{x}} u(x)=\sum_{n=0}^{\infty} \frac{z^{n}}{n !} \partial_{x}^{n} u(x)=u(x+z)
$$

for any analytic function $u(x)$, we have

$$
\begin{array}{r}
\int_{\mathbb{R}} v(t, x, d z)\left(u(x+z)-u(x)-z \partial_{x} u(x)\right) \\
=\int_{\mathbb{R}} v(t, x, d z)\left(e^{z \partial_{x}}-1-z \partial_{x}\right) u(x) .
\end{array}
$$

Then $\mathcal{A}$ can be represented as

$$
\mathcal{A}=\phi(t, x, \mathcal{D}), \quad \mathcal{D}=-i \partial_{x},
$$

since by (9) and (10)

$$
\begin{aligned}
\phi(t, x, \mathcal{D})= & \gamma(t, x)\left(\partial_{x}-1\right)+a(t, x)\left(\partial_{x}^{2}-\partial_{x}\right) \\
& -\int_{\mathbb{R}} v(t, x, d z)\left(e^{z}-1-z\right) \partial_{x}+\int_{\mathbb{R}} v(t, x, d z)\left(e^{z \partial_{x}}-1-z \partial_{x}\right) .
\end{aligned}
$$

If coefficients $a(t), \gamma(t), v(t, d z)$ are independent of $x$, then we have the usual characterization of $\mathcal{A}$ as a multiplication by $\phi$ operator in the Fourier space:

$$
\mathcal{A}=\mathcal{F}^{-1}(\phi(t, \cdot) \mathcal{F}), \quad \phi(t, \cdot):=\phi(t, x, \cdot),
$$

where $\mathcal{F}$ and $\mathcal{F}^{-1}$ denote the (direct) Fourier and inverse Fourier transform operators, respectively,

$$
\mathcal{F} f(\xi)=\hat{f}(\xi):=\frac{1}{\sqrt{2 \pi}} \int_{\mathbb{R}} e^{-i \xi x} f(x) d x, \quad \mathcal{F}^{-1} f(x)=\frac{1}{\sqrt{2 \pi}} \int_{\mathbb{R}} e^{i \xi x} f(\xi) d \xi .
$$

Moreover, if the coefficients $a, \gamma, v(d z)$ are independent of both $t$ and $x$, then $\mathcal{A}$ is the generator of a Lévy process $X$ and $\phi(\cdot):=\phi(t, x, \cdot)$ is the characteristic exponent of $X$ :

$$
\mathbb{E}\left[e^{i \xi X_{t}}\right]=e^{t \phi(\xi)}
$$


4. Density and option price expansions (a formal description). Our goal is to construct an approximate solution of Cauchy problem (6). We assume that the symbol of $\mathcal{A}$ admits an expansion of the form

$$
\phi(t, x, \xi)=\sum_{n=0}^{\infty} B_{n}(x) \phi_{n}(t, \xi),
$$

where $\phi_{n}(t, \xi)$ is of the form

$$
\begin{aligned}
\phi_{n}(t, \xi)= & \gamma_{n}(t)(i \xi-1)+a_{n}(t)\left(-\xi^{2}-i \xi\right) \\
& -\int_{\mathbb{R}} v_{n}(t, d z)\left(e^{z}-1-z\right) i \xi+\int_{\mathbb{R}} v_{n}(t, d z)\left(e^{i z \xi}-1-i z \xi\right)
\end{aligned}
$$

and $\left\{B_{n}\right\}_{n \geq 0}$ is some expansion basis with $B_{n}$ being an analytic function for each $n \geq 0$, and $B_{0} \equiv 1$ (see Examples 1,2 and 3 below). Note that $\phi_{n}(t, \xi)$ is the symbol of an operator

$$
\mathcal{A}_{n}:=\phi_{n}(t, \mathcal{D}), \quad \mathcal{D}=-i \partial_{x},
$$

so that

$$
\mathcal{A}_{n} \psi_{\xi}(x)=\phi_{n}(t, \xi) \psi_{\xi}(x) .
$$

Thus, formally the generator $\mathcal{A}$ can be written as follows:

$$
\mathcal{A}=\sum_{n=0}^{\infty} B_{n}(x) \mathcal{A}_{n} .
$$

Note that $\mathcal{A}_{0}$ is the generator of a time-dependent Lévy-type process $X^{(0)}$. In the time-independent case, $X^{(0)}$ is a Lévy process and $\phi_{0}(\cdot):=\phi_{0}(t, \cdot)$ is its characteristic exponent.

EXAMPLE 1 (Taylor series expansion). Pagliarani, Pascucci and Riga (2013) approximate the drift and diffusion coefficients of $\mathcal{A}$ as a power series about an arbitrary point $\bar{x} \in \mathbb{R}$. In our more general setting, this corresponds to setting $B_{n}(x)=(x-\bar{x})^{n}$ and expanding the diffusion and killing coefficients $a(t, \cdot)$ and $\gamma(t, \cdot)$, as well as the Lévy measure $v(t, \cdot, d z)$ as follows:

$$
\begin{aligned}
& a(t, x)=\sum_{n=0}^{\infty} a_{n}(t, \bar{x}) B_{n}(x), \quad a_{n}(t, \bar{x})=\frac{1}{n !} \partial_{x}^{n} a(t, \bar{x}), \\
& \gamma(t, x)=\sum_{n=0}^{\infty} \gamma_{n}(t, \bar{x}) B_{n}(x), \quad \gamma_{n}(t, \bar{x})=\frac{1}{n !} \partial_{x}^{n} \gamma(t, \bar{x}) \\
& v(t, x, d z)=\sum_{n=0}^{\infty} v_{n}(t, \bar{x}, d z) B_{n}(x), \quad v_{n}(t, \bar{x}, d z)=\frac{1}{n !} \partial_{x}^{n} \nu(t, \bar{x}, d z)
\end{aligned}
$$


In this case, (11) and (13) become (resp.)

$$
\phi(t, x, \xi)=\sum_{n=0}^{\infty}(x-\bar{x})^{n} \phi_{n}(t, \xi), \quad \mathcal{A}=\sum_{n=0}^{\infty}(x-\bar{x})^{n} \phi_{n}(t, \mathcal{D}),
$$

where, for all $n \geq 0$, the symbol $\phi_{n}(t, \xi)$ is given by (12) with coefficients given by (14). The choice of $\bar{x}$ is somewhat arbitrary. However, a convenient choice that seems to work well in most applications is to choose $\bar{x}$ near $X_{t}$, the current level of $X$. Hereafter, to simplify notation, when discussing implementation of the Taylor-series expansion, we suppress the $\bar{x}$-dependence: $a_{n}(t, \bar{x}) \rightarrow a_{n}(t)$, $\gamma_{n}(t, \bar{x}) \rightarrow \gamma_{n}(t)$ and $v_{n}(t, \bar{x}, d z) \rightarrow v_{n}(t, d z)$.

EXAMPLE 2 (Two-point Taylor series expansion). Suppose $f$ is an analytic function with domain $\mathbb{R}$ and $\bar{x}_{1}, \bar{x}_{2} \in \mathbb{R}$. Then the two-point Taylor series of $f$ is given by

$$
f(x)=\sum_{n=0}^{\infty}\left(c_{n}\left(\bar{x}_{1}, \bar{x}_{2}\right)\left(x-\bar{x}_{1}\right)+c_{n}\left(\bar{x}_{2}, \bar{x}_{1}\right)\left(x-\bar{x}_{2}\right)\right)\left(x-\bar{x}_{1}\right)^{n}\left(x-\bar{x}_{2}\right)^{n},
$$

where

$$
\begin{aligned}
& c_{0}\left(\bar{x}_{1}, \bar{x}_{2}\right)=\frac{f\left(\bar{x}_{2}\right)}{\bar{x}_{2}-\bar{x}_{1}}, \\
& c_{n}\left(\bar{x}_{1}, \bar{x}_{2}\right)=\sum_{k=0}^{n} \frac{(k+n-1) !}{k ! n !(n-k) !} \frac{(-1)^{k} k \partial_{\bar{x}_{1}}^{n-k} f\left(\bar{x}_{1}\right)+(-1)^{n+1} n \partial_{\bar{x}_{2}}^{n-k} f\left(\bar{x}_{2}\right)}{\left(\bar{x}_{1}-\bar{x}_{2}\right)^{k+n+1}} .
\end{aligned}
$$

For the derivation of this result, we refer the reader to Estes and Lancaster (1972), López and Temme (2002). Note truncating the two-point Taylor series expansion (15) at $n=m$ results in an expansion which of $f$ which is of order $\mathcal{O}\left(x^{2 n+1}\right)$.

The advantage of using a two-point Taylor series is that, by considering the first $n$ derivatives of a function $f$ at two points $\bar{x}_{1}$ and $\bar{x}_{2}$, one can achieve a more accurate approximation of $f$ over a wider range of values than if one were to approximate $f$ using $2 n$ derivatives at a single point (i.e., the usual Taylor series approximation).

If we associate expansion (15) with an expansion of the form $f(x)=$ $\sum_{n=0}^{\infty} f_{n} B_{n}(x)$ then $f_{0} B_{0}(x)=c_{n}\left(\bar{x}_{1}, \bar{x}_{2}\right)\left(x-\bar{x}_{1}\right)+c_{n}\left(\bar{x}_{2}, \bar{x}_{1}\right)\left(x-\bar{x}_{2}\right)$, which is affine in $x$. Thus, the terms in the two-point Taylor series expansion would not be a suitable basis in (11) since $B_{0}(x) \neq 1$. However, one can always introduce a constant $M$ and define a function

$$
F(x):=f(x)-M \quad \text { so that } f(x)=M+F(x) .
$$

Then one can express $f$ as

$$
\begin{aligned}
f(x)=M+\sum_{n=1}^{\infty} & \left(C_{n-1}\left(\bar{x}_{1}, \bar{x}_{2}\right)\left(x-\bar{x}_{1}\right)+C_{n-1}\left(\bar{x}_{2}, \bar{x}_{1}\right)\left(x-\bar{x}_{2}\right)\right) \\
& \times\left(x-\bar{x}_{1}\right)^{n-1}\left(x-\bar{x}_{2}\right)^{n-1},
\end{aligned}
$$


where the $C_{n}$ are as given in (16) with $f \rightarrow F$. If we associate expansion (18) with an expansion of the form $f(x)=\sum_{n=0}^{\infty} f_{n} B_{n}(x)$, then we see that $f_{0} B_{0}(x)=M$ and one can choose $B_{0}(x)=1$. Thus, as written in (18), the terms of the two-point Taylor series can be used as a suitable basis in (11).

Consider the following case: suppose $a(t, x), \gamma(t, x)$ and $v(t, x, d z)$ are of the form

$$
\begin{aligned}
a(t, x) & =f(x) A(t), \quad \gamma(t, x)=f(x) \Gamma(t), \\
v(t, x, d z) & =f(x) \mathcal{N}(t, d z),
\end{aligned}
$$

so that $\phi(t, x, \xi)=f(x) \Phi(t, \xi)$ with

$$
\begin{aligned}
\Phi(t, \xi)= & \Gamma(t)(i \xi-1)+A(t)\left(-\xi^{2}-i \xi\right) \\
& -\int_{\mathbb{R}} \mathcal{N}(t, d z)\left(e^{z}-1-z\right) i \xi+\int_{\mathbb{R}} \mathcal{N}(t, d z)\left(e^{i \xi z}-1-i \xi z\right) .
\end{aligned}
$$

It is certainly plausible that the symbol of $\mathcal{A}$ would have such a form since, from a modeling perspective, it makes sense that default intensity, volatility and jump-intensity would be proportional. Indeed, the Jump-to-Default CEV model (JDCEV) of Carr and Linetsky (2006), Carr and Madan (2010) has a similar restriction on the form of the drift, volatility and killing coefficients.

Now, under the dynamics of (19), observe that $\phi(t, x, \xi)$ and $\mathcal{A}$ can be written as in (11) and (13), respectively, with $B_{0}=1$ and

$$
\begin{aligned}
B_{n}(x)= & \left(C_{n-1}\left(\bar{x}_{1}, \bar{x}_{2}\right)\left(x-\bar{x}_{1}\right)+C_{n-1}\left(\bar{x}_{2}, \bar{x}_{1}\right)\left(x-\bar{x}_{2}\right)\right) \\
& \times\left(x-\bar{x}_{1}\right)^{n-1}\left(x-\bar{x}_{2}\right)^{n-1}, \quad n \geq 1 .
\end{aligned}
$$

As above $C_{n}$ (capital "C") are given by (16) with $f \rightarrow F:=f-M$ and

$$
\phi_{0}(t, \xi)=M \Phi(t, \xi), \quad \phi_{n}(t, \xi)=\Phi(t, \xi), \quad n \geq 1 .
$$

As in Example 1, the choice of $\bar{x}_{1}, \bar{x}_{2}$ and $M$ is somewhat arbitrary. But a choice that seems to work well is to set $\bar{x}_{1}=X_{t}-\Delta$ and $\bar{x}_{2}=X_{t}+\Delta$ where $\Delta>0$ is a constant and $M=f\left(X_{t}\right)$. It is also a good idea to check that, for a given choice of $\bar{x}_{1}$ and $\bar{x}_{2}$, the two-point Taylor series expansion provides a good approximation of $f$ in the region of interest.

Note we assumed the form (19) only for sake of simplicity. Indeed, the general case can be accommodated by suitably extending expansion (11) to the more general form

$$
\phi(t, x, \xi)=\sum_{n=0}^{\infty} \sum_{i=1}^{3} B_{i, n}(x) \phi_{i, n}(t, \xi),
$$

where $\phi_{i, n}$ for $i=1,2,3$ are related to the diffusion, jump and default symbols, respectively. For brevity, however, we omit the details of the general case. 
EXAMPLE 3 (Nonlocal approximation in weighted $L^{2}$-spaces). Suppose $\left\{B_{n}\right\}_{n \geq 0}$ is a fixed orthonormal basis in some (possibly weighted) space $L^{2}(\mathbb{R}$, $\mathfrak{m}(x) \bar{d} x)$ and that $\phi(t, \cdot, \xi) \in L^{2}(\mathbb{R}, \mathfrak{m}(x) d x)$ for all $(t, \xi)$. Then we can represent $\phi(t, x, \xi)$ in the form (11) where now the $\left\{\phi_{n}\right\}_{n \geq 0}$ are given by

$$
\phi_{n}(t, \xi)=\left\langle B_{n}(\cdot), \phi(t, \cdot, \xi)\right\rangle_{\mathfrak{m}}, \quad n \geq 0 .
$$

A typical example would be to choose Hermite polynomials $H_{n}$ centered at $\bar{x}$ as basis functions, which (as normalized below) are orthonormal under a Gaussian weighting

$$
B_{n}(x)=H_{n}(x-\bar{x}), \quad H_{n}(x):=\frac{1}{\sqrt{(2 n) ! ! \sqrt{\pi}}} \frac{\partial_{x}^{n} \exp \left(-x^{2}\right)}{\exp \left(-x^{2}\right)}, \quad n \geq 0 .
$$

In this case, we have

$$
\begin{aligned}
\phi_{n}(t, \xi)=\left\langle\phi(t, \cdot, \xi), B_{n}\right\rangle_{\mathfrak{m}}:=\int_{\mathbb{R}} \phi(t, x, \xi) & B_{n}(x) \mathfrak{m}(x) d x \\
\mathfrak{m}(x) & :=\exp \left(-(x-\bar{x})^{2}\right) .
\end{aligned}
$$

Once again, the choice of $\bar{x}$ is arbitrary. But it is logical to choose $\bar{x}$ near $X_{t}$, the present level of the underlying $X$. Note that, in the case of an $L^{2}$ orthonormal basis, differentiability of the coefficients $(a(t, \cdot), \gamma(t, \cdot), v(t, \cdot, d z))$ is not required. This is a significant advantage over the Taylor and two-point Taylor basis functions considered in Examples 1 and 2, which do require differentiability of the coefficients.

Now, returning to Cauchy problem (6), we suppose that $v=v(t, x)$ can be written as follows:

$$
v=\sum_{n=0}^{\infty} v_{n}
$$

Following Pagliarani, Pascucci and Riga (2013), we insert expansions (13) and (22) into Cauchy problem (6) and find

$$
\begin{array}{ll}
\left(\partial_{t}+\mathcal{A}_{0}\right) v_{0}=0, \quad v_{0}(T, x)=h(x), & \\
\left(\partial_{t}+\mathcal{A}_{0}\right) v_{n}=-\sum_{k=1}^{n} B_{k}(x) \mathcal{A}_{k} v_{n-k}, & v_{n}(T, x)=0 .
\end{array}
$$

We are now in a position to find the explicit expression for $\widehat{v}_{n}$, the Fourier transform of $v_{n}$ in (23)-(24).

THEOREM 1. Suppose $h \in L^{1}(\mathbb{R}, d x)$ and let $\widehat{h}$ denote its Fourier transform. Suppose further that $v_{n}$ and its Fourier transform $\widehat{v}_{n}$ exist, and that both the left- 
and right-hand side of (23)-(24) belong to $L^{1}(\mathbb{R}, d x)$. Then $\widehat{v}_{n}(t, \xi)$ is given by

$$
\begin{aligned}
& \widehat{v}_{0}(t, \xi)=\exp \left(\int_{t}^{T} \phi_{0}(s, \xi) d s\right) \widehat{h}(\xi) \\
& \widehat{v}_{n}(t, \xi)=\sum_{k=1}^{n} \int_{t}^{T} \exp \left(\int_{t}^{s} \phi_{0}(u, \xi) d u\right) B_{k}\left(i \partial_{\xi}\right) \phi_{k}(s, \xi) \widehat{v}_{n-k}(s, \xi) d s
\end{aligned}
$$

$$
n \geq 1 \text {. }
$$

Note that the operator $B_{k}\left(i \partial_{\xi}\right)$ acts on everything to the right of it.

Proof. See Appendix A.

REMARK 4. To compute survival probabilities $v(t, x)=v(t, x ; T)$ over the interval $[t, T]$, one assumes a payoff function $h(x)=1$. Note that the Fourier transform of a constant is simply a Dirac delta function: $\widehat{h}(\xi)=\delta(\xi)$. Thus, when computing survival probabilities, (possibly defaultable) bond prices and credit spreads, no numerical integration is required. Rather, one simply uses the following identity:

$$
\int_{\mathbb{R}} \widehat{u}(\xi) \partial_{\xi}^{n} \delta(\xi) d \xi=\left.(-1)^{n} \partial_{\xi}^{n} \widehat{u}(\xi)\right|_{\xi=0}
$$

to compute inverse Fourier transforms.

REMARK 5. Assuming $\widehat{v}_{n} \in L^{1}(\mathbb{R}, d x)$, one recovers $v_{n}$ using

$$
v_{n}(t, x)=\int_{\mathbb{R}} d \xi \frac{1}{\sqrt{2 \pi}} e^{i \xi x} \widehat{v}_{n}(t, \xi) .
$$

As previously mentioned, to obtain the FK transition densities $p(t, x ; T, y)$ one simply sets $h(x)=\delta_{y}(x)$. In this case, $\widehat{h}(\xi)$ becomes $\psi_{y}(-\xi)$.

When the coefficients $(a, \gamma, v)$ are time-homogeneous, then the results of Theorem 1 simplify considerably, as we show in the following corollary.

COROLlary 1 (Time-homogeneous case). Suppose that $X$ has timehomogeneous dynamics with the local variance, default intensity and Lévy measure given by $a(x), \gamma(x)$ and $\nu(x, d z)$, respectively. Then the symbol $\phi_{n}(t, \xi)=\phi_{n}(\xi)$ is independent of $t$. Define

$$
\tau(t):=T-t .
$$

Then, for $n \leq 0$ we have

$$
v_{n}(t, x)=u_{n}(\tau(t), x)
$$


where

$$
\begin{aligned}
& \widehat{u}_{0}(\tau, \xi)=e^{\tau \phi_{0}(\xi)} \widehat{h}(\xi) \\
& \widehat{u}_{n}(\tau, \xi)=\sum_{k=1}^{n} \int_{0}^{\tau} e^{(\tau-s) \phi_{0}(\xi)} B_{k}\left(i \partial_{\xi}\right) \phi_{k}(\xi) \widehat{u}_{n-k}(s, \xi) d s, \quad n \geq 1
\end{aligned}
$$

PROOF. The proof is an algebraic computation. For brevity, we omit the details.

EXAMPLE 4. Consider the Taylor density expansion of Example 1. That is, $B_{n}(x)=(x-\bar{x})^{n}$. Then, in the time-homogeneous case, we find that $\widehat{u}_{1}(t, \xi)$ and $\widehat{u}_{2}(t, \xi)$ are given explicitly by

$$
\begin{aligned}
\widehat{u}_{1}(t, \xi)=e^{t \phi_{0}(\xi)}( & t \widehat{h}(\xi) \bar{x} \phi_{1}(\xi)+i t \phi_{1}(\xi) \widehat{h}^{\prime}(\xi)+\frac{1}{2} i t^{2} \widehat{h}(\xi) \phi_{1}(\xi) \phi_{0}^{\prime}(\xi) \\
\widehat{u}_{2}(t, \xi)=e^{t \phi_{0}(\xi)}\left(\frac{1}{2} t^{2} \widehat{h}(\xi) \bar{x}^{2} \phi_{1}^{2}(\xi)+t \widehat{h}(\xi) \phi_{1}^{\prime}(\xi)\right) & -2 i t \bar{x} \phi_{2}(\xi) \widehat{h}^{\prime}(\xi) \\
& -\frac{1}{2} i t^{3} \widehat{h}(\xi) \bar{x} \phi_{1}^{2}(\xi) \phi_{0}^{\prime}(\xi)-i t^{2} \widehat{h}(\xi) \bar{x} \phi_{2}^{2}(\xi) \widehat{h}^{\prime}(\xi) \\
& -\frac{1}{2} t^{3} \phi_{1}(\xi)^{2} \widehat{h}^{\prime}(\xi) \phi_{0}^{\prime}(\xi)-t^{2} \phi_{2}(\xi) \widehat{h}^{\prime}(\xi) \phi_{0}^{\prime}(\xi) \\
& -\frac{1}{8} t^{4} \widehat{h}(\xi) \phi_{1}^{2}(\xi)\left(\phi_{0}^{\prime}(\xi)\right)^{2}-\frac{1}{3} t^{3} \widehat{h}(\xi) \phi_{2}(\xi)\left(\phi_{0}^{\prime}(\xi)\right)^{2} \\
& -\frac{3}{2} i t^{2} \widehat{h}(\xi) \bar{x} \phi_{1}(\xi) \phi_{1}^{\prime}(\xi) \\
& -\frac{3}{2} t^{2} \phi_{1}(\xi) \widehat{h}^{\prime}(\xi) \phi_{1}^{\prime}(\xi)-\frac{2}{3} t^{3} \widehat{h}(\xi) \phi_{1}(\xi) \phi_{0}^{\prime}(\xi) \phi_{1}^{\prime}(\xi) \\
& -\frac{1}{2} t^{2} \widehat{h}(\xi)\left(\phi_{1}^{\prime}(\xi)\right)^{2}-2 i t \widehat{h}(\xi) \bar{x} \phi_{2}^{\prime}(\xi) \\
& -2 t \widehat{h}^{\prime}(\xi) \phi_{2}^{\prime}(\xi)-t^{2} \widehat{h}(\xi) \phi_{0}^{\prime}(\xi) \phi_{2}^{\prime}(\xi)-\frac{1}{2} t^{2} \phi_{1}(\xi)^{2} \widehat{h}^{\prime \prime}(\xi) \\
& -t \phi_{2}(\xi) \widehat{h}^{\prime \prime}(\xi)-\frac{1}{6} t^{3} \widehat{h}(\xi) \phi_{1}^{2}(\xi) \phi_{0}^{\prime \prime}(\xi) \\
& \left.-\frac{1}{2} t^{2} \widehat{h}(\xi) \phi_{2}(\xi) \phi_{0}^{\prime \prime}(\xi)-\frac{1}{2} t^{2} \widehat{h}(\xi) \phi_{1}(\xi) \phi_{1}^{\prime \prime}(\xi)-t \widehat{h}(\xi) \phi_{2}^{\prime \prime}(\xi)\right) .
\end{aligned}
$$

Higher order terms are quite long. However, they can be computed quickly and explicitly using the Mathematica code provided in Appendix B. The code in the Appendix can be easily modified for use with other basis functions.

REMARK 6. As in Pagliarani, Pascucci and Riga (2013), when considering models with Gaussian-type jumps, that is, models with a state-dependent Lévy measure $v(t, x, d z)$ of the form (28) below, all terms in the expansion for the transition density become explicit. Likewise, for models with Gaussian-type jumps, all terms in the expansion for the price of an option are also explicit, assuming the payoff is integrable against Gaussian functions. 
REMARK 7. Many common payoff functions (e.g., calls and puts) are not integrable: $h \notin L^{1}(\mathbb{R}, d x)$. Such payoffs may sometimes be accommodated using generalized Fourier transforms. Assume

$$
\widehat{h}(\xi):=\int_{\mathbb{R}} d x \frac{1}{\sqrt{2 \pi}} e^{-i \xi x} h(x)<\infty \quad \text { for some } \xi=\xi_{r}+i \xi_{i} \text { with } \xi_{r}, \xi_{i} \in \mathbb{R} .
$$

Assume also that $\phi\left(t, x, \xi_{r}+i \xi_{i}\right)$ is analytic as a function of $\xi_{r}$. Then the formulas appearing in Theorem 1 and Corollary 1 are valid and integration in (27) is with respect to $\xi_{r}$ (i.e., $d \xi \rightarrow d \xi_{r}$ ). For example, the payoff of a European call option with payoff function $h(x)=\left(e^{x}-e^{k}\right)^{+}$has a generalized Fourier transform

$$
\begin{aligned}
\widehat{h}(\xi)=\int_{\mathbb{R}} d x \frac{1}{\sqrt{2 \pi}} e^{-i \xi x}\left(e^{x}-e^{k}\right)^{+} & =\frac{-e^{k-i k \xi}}{\sqrt{2 \pi}\left(i \xi+\xi^{2}\right)}, \\
\xi & =\xi_{r}+i \xi_{i}, \xi_{r} \in \mathbb{R}, \xi_{i} \in(-\infty,-1) .
\end{aligned}
$$

In any practical scenario, one can only compute a finite number of terms in (22). Thus, we define $v^{(N)}$, the $N$ th order approximation of $v$ by

$$
\begin{aligned}
v^{(N)}(t, x) & =\sum_{n=0}^{N} v_{n}(t, x)=\int_{\mathbb{R}} d \xi \frac{1}{\sqrt{2 \pi}} e^{i \xi x} \widehat{v}^{(n)}(t, \xi), \\
\widehat{v}^{(N)}(t, \xi): & =\sum_{n=0}^{N} \widehat{v}_{n}(t, \xi) .
\end{aligned}
$$

The function $u^{(N)}(t, x)$ (which we use for time-homogeneous cases) and the approximate FK transition density $p^{(N)}(t, x ; T, y)$ are defined in an analogous fashion.

5. Pointwise error bounds for Gaussian models. In this section, we state some pointwise error estimates for $p^{(N)}(t, x ; T, y)$, the $N$ th order approximation of the FK density of $\left(\partial_{t}+\mathcal{A}\right)$ with $\mathcal{A}$ as in (7). Throughout this section, we assume Gaussian-type jumps with $(t, x)$-dependent mean, variance and jump intensities. Furthermore, we work specifically with the Taylor series expansion of Example 1. That is, we use basis functions $B_{n}(x)=(x-\bar{x})^{n}$.

THEOREM 2. Assume that

$$
m \leq a(t, x) \leq M, \quad 0 \leq \gamma(t, x) \leq M, \quad t \in[0, T], x \in \mathbb{R},
$$

for some positive constants $m$ and $M$, and that

$$
\begin{aligned}
v(t, x, d z) & =\lambda(t, x) \mathcal{N}_{\mu(t, x), \delta^{2}(t, x)}(d z) \\
& :=\frac{\lambda(t, x)}{\sqrt{2 \pi} \delta(t, x)} e^{-(z-\mu(t, x))^{2} /\left(2 \delta^{2}(t, x)\right)} d z
\end{aligned}
$$


with

$$
m \leq \delta^{2}(t, x) \leq M, \quad 0 \leq \lambda(t, x),|\mu(t, x)| \leq M, \quad t \in[0, T], x \in \mathbb{R} .
$$

Moreover assume that $a, \gamma, \lambda, \delta, \mu$ and their $x$-derivatives are bounded and Lipschitz continuous in $x$, and uniformly bounded with respect to $t \in[0, T]$. Let $\bar{x}=y$ in (14). Then, for $N \geq 1$, we have

$$
\begin{aligned}
& \quad\left|p(t, x ; T, y)-p^{(N)}(t, x ; T, y)\right| \\
& \quad \leq g_{N}(T-t)\left(\bar{\Gamma}(t, x ; T, y)+\left\|\partial_{x} \nu\right\|_{\infty} \widetilde{\Gamma}(t, x ; T, y)\right),
\end{aligned}
$$

for any $x, y \in \mathbb{R}$ and $t<T$, where

$$
g_{N}(s)=\mathcal{O}(s) \quad \text { as } s \rightarrow 0^{+} .
$$

Here, the function $\bar{\Gamma}$ is the fundamental solution of the constant coefficients jumpdiffusion operator

$$
\partial_{t} u(t, x)+\frac{\bar{M}}{2} \partial_{x x}+\bar{M} \int_{\mathbb{R}}(u(t, x+z)-u(t, x)) \mathcal{N}_{\bar{M}, \bar{M}}(d z),
$$

where $\bar{M}$ is a suitably large constant, and $\widetilde{\Gamma}$ is defined as

$$
\widetilde{\Gamma}(t, x ; T, y)=\sum_{k=0}^{\infty} \frac{\bar{M}^{k / 2}(T-t)^{k / 2}}{\sqrt{k !}} \mathrm{C}^{k+1} \bar{\Gamma}(t, x ; T, y),
$$

and where $\mathcal{C}$ is the convolution operator acting as

$$
\mathcal{C} f(x)=\int_{\mathbb{R}} f(x+z) \mathcal{N}_{\bar{M}, \bar{M}}(d z) .
$$

ProOF. An outline of the proof is provided in Appendix C. For a detailed proof, we refer to Lorig, Pagliarani and Pascucci (2013).

REMARK 8. The functions $\mathcal{C}^{k} \bar{\Gamma}$ take the following form:

$\mathrm{C}^{k} \bar{\Gamma}(t, x ; T, y)$

(31) $=e^{-\bar{M}(T-t)} \sum_{n=0}^{\infty} \frac{(\bar{M}(T-t))^{n}}{n ! \sqrt{2 \pi \bar{M}(T-t+n+k)}} \exp \left(-\frac{(x-y+\bar{M}(n+k))^{2}}{2 \bar{M}(T-t+n+k)}\right)$,

$$
k \geq 0
$$

\footnotetext{
${ }^{5}$ Here, $\left\|\partial_{x} v\right\|_{\infty}:=\max \left\{\left\|\partial_{x} \lambda\right\|_{\infty},\left\|\partial_{x} \delta\right\|_{\infty},\left\|\partial_{x} \mu\right\|_{\infty}\right\}$, where $\|\cdot\|_{\infty}$ denotes the sup-norm on $(0, T) \times \mathbb{R}$. Note that $\left\|\partial_{x} \nu\right\|_{\infty}=0$ if $\lambda, \delta, \mu$ are constants.
} 
and, therefore, $\widetilde{\Gamma}$ can be explicitly written as

$$
\begin{aligned}
\widetilde{\Gamma}(t, x ; T, y)=e^{-\bar{M}(T-t)} \sum_{n, k=0}^{\infty} & \frac{(\bar{M}(T-t))^{n+k / 2}}{n ! \sqrt{k !} \sqrt{2 \pi \bar{M}(T-t+n+k+1)}} \\
& \times \exp \left(-\frac{(x-y+\bar{M}(n+k+1))^{2}}{2 \bar{M}(T-t+n+k+1)}\right) .
\end{aligned}
$$

By Remark 8, it follows that, when $k=0$ and $x \neq y$, the asymptotic behavior as $t \rightarrow T$ of the sum in (31) depends only on the $n=1$ term. Consequently, we have $\bar{\Gamma}(t, x ; T, y)=\mathcal{O}(T-t)$ as $(T-t)$ tends to 0 . On the other hand, for $k \geq 1$, $\mathcal{C}^{k} \bar{\Gamma}(t, x ; T, y)$, and thus also $\widetilde{\Gamma}(t, x ; T, y)$, tends to a positive constant as $(T-t)$ goes to 0 . It is then clear by (29) that, with $x \neq y$ fixed, the asymptotic behavior of the error, when $t$ tends to $T$, changes from $(T-t)$ to $(T-t)^{2}$ depending on whether the Lévy measure is locally-dependent or not.

Theorem 2 extends the previous results in Pagliarani, Pascucci and Riga (2013) where only the purely diffusive case (i.e., $\lambda \equiv 0$ ) is considered. In that case, an estimate analogous to (29) holds with

$$
g_{N}(s)=\mathcal{O}\left(s^{(N+1) / 2}\right) \quad \text { as } s \rightarrow 0^{+} .
$$

Theorem 2 shows that for jump processes, increasing the order of the expansion for $N$ greater than one, theoretically does not give any gain in the rate of convergence of the asymptotic expansion as $t \rightarrow T^{-}$; this is due to the fact that the expansion is based on a local (Taylor) approximation while the PIDE contains a nonlocal part. This estimate is in accord with the results in Benhamou, Gobet and Miri (2009) where only the case of constant Lévy measure is considered. Thus, Theorem 2 extends the latter results to state dependent Gaussian jumps using a completely different technique. Extensive numerical tests showed that the firstorder approximation gives extremely accurate results and the precision seems to be further improved by considering higher order approximations.

COROLLARY 2. Under the assumptions of Theorem 2, we have the following estimate for the error on the approximate prices:

$$
\begin{aligned}
& \left|v(t, x)-v^{(N)}(t, x)\right| \\
& \quad \leq g_{N}(T-t) \int_{\mathbb{R}}|h(y)|\left(\bar{\Gamma}(t, x ; T, y)+\left\|\partial_{x} v\right\|_{\infty} \widetilde{\Gamma}(t, x ; T, y)\right) d y,
\end{aligned}
$$

for any $x \in \mathbb{R}$ and $t<T$.

Some possible extensions of these asymptotic error bounds to general Lévy measures are possible, though they are certainly not straightforward. Indeed, the proof of Theorem 2 is based on some pointwise uniform estimates for the fundamental solution of the constant coefficient operator, that is, the transition density of 
a compound Poisson process with Gaussian jumps. When considering other Lévy measures these estimates would be difficult to carry out, especially in the case of jumps with infinite activity, but they might be obtained in some suitable normed functional space. This might lead to error bounds for short maturities, which are expressed in terms of a suitable norm, as opposed to uniform pointwise bounds.

REMARK 9. Since, in general, it is hard to derive the truncation error bound, the reader may wonder how to determine the number of terms to include in the asymptotic expansion. Though we provide a general expression for the $n$th term, realistically, only the fourth-order term can be computed. That said, in practice, three terms provide an approximation which is accurate enough for most applications (i.e., the resulting approximation error is smaller than the bid-ask spread typically quoted on the market). Since $v^{(n)}$ only requires a single Fourier integration, there is no numerical advantage for choosing smaller $n$. As such, for financial applications we suggest using $n=3$ or $n=4$.

6. Examples. In this section, in order to illustrate the versatility of our asymptotic expansion, we apply our approximation technique to a variety of different Lévy-type models. We consider both finite and infinite activity Lévy-type measures and models with and without a diffusion component. We study not only option prices, but also implied volatilities. In each setting, if the exact or approximate option price has been computed by a method other than our own, we compare this to the option price obtained by our approximation. For cases where the exact or approximate option price is not analytically available, we use Monte Carlo methods to verify the accuracy of our method.

Note that, some of the examples considered below do not satisfy the conditions listed in Section 2. In particular, we will consider coefficients $(a, \gamma, v)$ that are not bounded. Nevertheless, the formal results of Section 4 work well in the examples considered.

6.1. CEV-like Lévy-type processes. We consider a Lévy-type process of the form (1) with CEV-like volatility and jump-intensity. Specifically, the log-price dynamics are given by

$$
\begin{array}{ll}
a(x)=\frac{1}{2} \delta^{2} e^{2(\beta-1) x}, \quad v(x, d z)=e^{2(\beta-1) x} \mathcal{N}(d z), & \gamma(x)=0, \\
& \delta \geq 0, \beta \in[0,1],
\end{array}
$$

where $\mathcal{N}(d x)$ is a Lévy measure. When $\mathcal{N} \equiv 0$, this model reduces to the CEV model of Cox (1975). Note that, with $\beta \in[0,1)$, the volatility and jump-intensity increase as $x \rightarrow-\infty$, which is consistent with the leverage effect (i.e., a decrease in the value of the underlying is often accompanied by an increase in volatility/jump intensity). This characterization will yield a negative skew in the induced implied volatility surface. As the class of models described by (32) is of the form 
(19) with $f(x)=e^{2(\beta-1) x}$, this class naturally lends itself to the two-point Taylor series approximation of Example 2. Thus, for certain numerical examples in this section, we use basis functions $B_{n}$ given by (20). In this case, we choose expansion points $\bar{x}_{1}$ and $\bar{x}_{2}$ in a symmetric interval around $X_{0}$ and in (17) we choose $M=f\left(X_{0}\right)=e^{2(\beta-1) X_{0}}$. For other numerical examples, we use the (usual) onepoint Taylor series expansion $B_{n}(x)=(x-\bar{x})^{n}$. In this cases, we choose $\bar{x}=X_{0}$.

We will consider two different characterizations of $\mathcal{N}(d z)$ :

$$
\begin{aligned}
\text { Gaussian: } \mathcal{N}(d z) & =\lambda \frac{1}{\sqrt{2 \pi \eta^{2}}} \exp \left(\frac{-(z-m)^{2}}{2 \eta^{2}}\right) d z, \\
\text { Variance-Gamma: } \mathcal{N}(d z) & =\left(\frac{e^{-\lambda-|z|}}{\kappa|z|} \mathbb{I}_{\{z<0\}}+\frac{e^{-\lambda+z}}{\kappa z} \mathbb{I}_{\{z>0\}}\right) d z, \\
\lambda_{ \pm} & =\left(\sqrt{\frac{\theta^{2} \kappa^{2}}{4}+\frac{\rho^{2} \kappa}{2}} \pm \frac{\theta \kappa}{2}\right)^{-1} .
\end{aligned}
$$

Note that the Gaussian measure is an example of a finite-activity Lévy measure [i.e., $\mathcal{N}(\mathbb{R})<\infty$ ], whereas the Variance-Gamma measure, due to Madan, Carr and Chang (1998), is an infinite-activity Lévy measure [i.e., $\mathcal{N}(\mathbb{R})=\infty$ ]. As far as the authors of this paper are aware, there is no closed-form expression for option prices (or the transition density) in the setting of (32), regardless of the choice of $\mathcal{N}(d z)$. As such, we will compare our pricing approximation to prices of options computed via standard Monte Carlo methods.

REMARK $10{ }^{6}$ Note the CEV model typically includes an absorbing boundary condition at $S=0$. A more rigorous way to deal with degenerate dynamics, as in the CEV model, would be to approximate the solution of the Cauchy problem related to the process $S_{t}$ (as apposed to $X_{t}=\log S_{t}$ ). One would then equip the Cauchy problem with suitable Dirichlet conditions on the boundary $s=0$, and work directly in the variable $s \in \mathbb{R}_{+}$as opposed to the log-price on $x \in \mathbb{R}$. Indeed, this is the approach followed by Hagan and Woodward (1999) who approximate the true density $p$ by a Gaussian density $p_{0}$ through a heat kernel expansion: note that the supports of $p$ and $p_{0}$ are $\mathbb{R}_{+}$and $\mathbb{R}$, respectively. In order to take into account of the boundary behavior of the true density $p$, an improved approximation could be achieved by using the Green function of the heat operator for $\mathbb{R}_{+}$instead of the Gaussian kernel: this will be object of further research in a forthcoming paper.

\footnotetext{
${ }^{6} \mathrm{We}$ would like to thank an anonymous referee for bringing the issue of boundary conditions to our attention.
} 
We would also like to remark explicitly that our methodology is very general and works with different choices for the leading operator of the expansion, such as the constant-coefficient PIDEs we consider in the case of jumps. Nevertheless, in the present paper, when purely diffusive models are considered, we always take the heat operator as the leading term of our expansion. The main reasons are that (i) the heat kernel is convenient for its computational simplicity and (ii) the heat kernel allows for the possibility of passing directly from a Black-Scholes-type price expansion to an implied vol expansion.

6.1.1. Gaussian Lévy measure. In our first numerical experiment, we consider the case of Gaussian jumps. That is, $\mathcal{N}(d z)$ is given by (33). We fix the following parameters:

$$
\begin{aligned}
& \delta=0.20, \quad \beta=0.25, \quad \lambda=0.3, \quad m=-0.1, \\
& \eta=0.4, \quad S_{0}=e^{x}=1 .
\end{aligned}
$$

Using Corollary 1 , we compute the approximate prices $u^{(0)}(t, x ; K)$ and $u^{(3)}(t, x$; $K$ ) of a series of European puts over a range of strikes $K$ and with times to maturity $t=\{0.25,1.00,3.00,5.00\}$ (we add the parameter $K$ to the arguments of $u^{(n)}$ to emphasize the dependence of $u^{(n)}$ on the strike price $\left.K\right)$. To compute $u^{(i)}(t, x ; K)$, $i=\{0,3\}$ we use the we the usual one-point Taylor series expansion (Example 1). We also compute the price $u(t, x ; K)$ of each put by Monte Carlo simulation. For the Monte Carlo simulation, we use a standard Euler scheme with a time-step of $10^{-3}$ years, and we simulate $10^{6}$ sample paths. We denote by $u^{(\mathrm{MC})}(t, x ; K)$ the price of a put obtained by Monte Carlo simulation. As prices are often quoted in implied volatilities, we convert prices to implied volatilities by inverting the Black-Scholes formula numerically. That is, for a given put price $u(t, x ; K)$, we find $\sigma(t, K)$ such that

$$
u(t, x ; K)=u^{\mathrm{BS}}(t, x ; K, \sigma(t, K)),
$$

where $u^{\mathrm{BS}}(t, x ; K, \sigma)$ is the Black-Scholes price of the put as computed assuming a Black-Scholes volatility of $\sigma$. For convenience, we introduce the notation

$$
\operatorname{IV}[u(t, x ; K)]:=\sigma(t, K)
$$

to indicate the implied volatility induced by option price $u(t, x ; K)$. The results of our numerical experiments are plotted in Figure 1. We observe that $\operatorname{IV}\left[u^{(3)}(t, x ; K)\right]$ agrees almost exactly with $\operatorname{IV}\left[u^{(\mathrm{MC})}(t, x ; K)\right]$. The computed prices $u^{(3)}(t, x ; K)$ and their induced implied volatilities $\operatorname{IV}\left[u^{(3)}(t, x ; K)\right]$, as well as $95 \%$ confidence intervals resulting from the Monte Carlo simulations can be found in Table 1. 
$t=0.25$
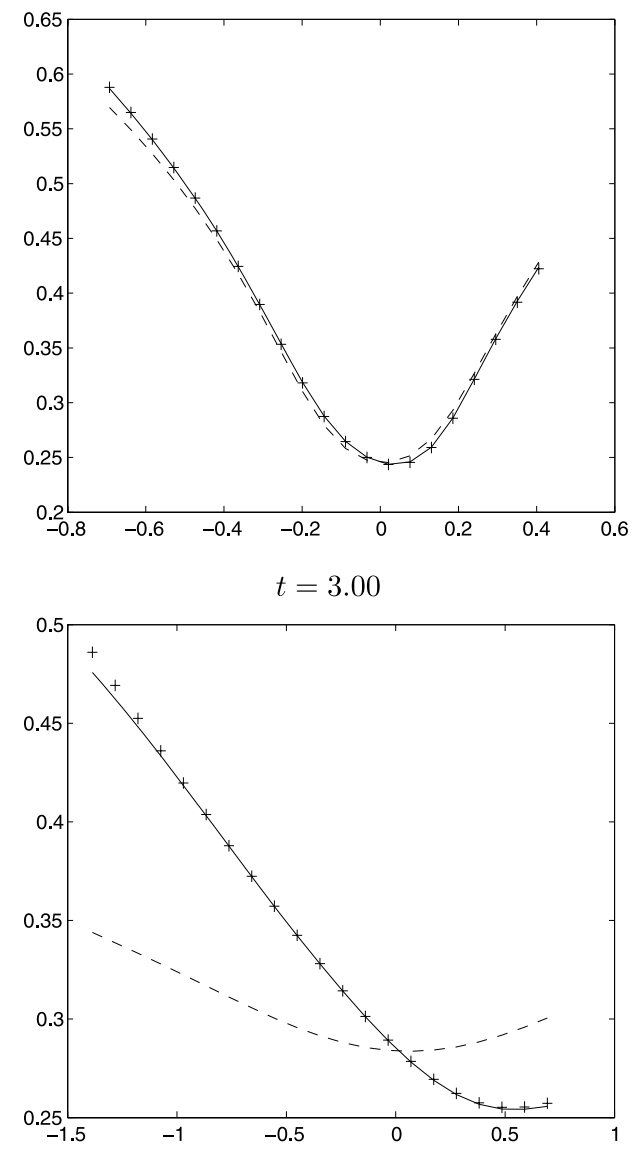
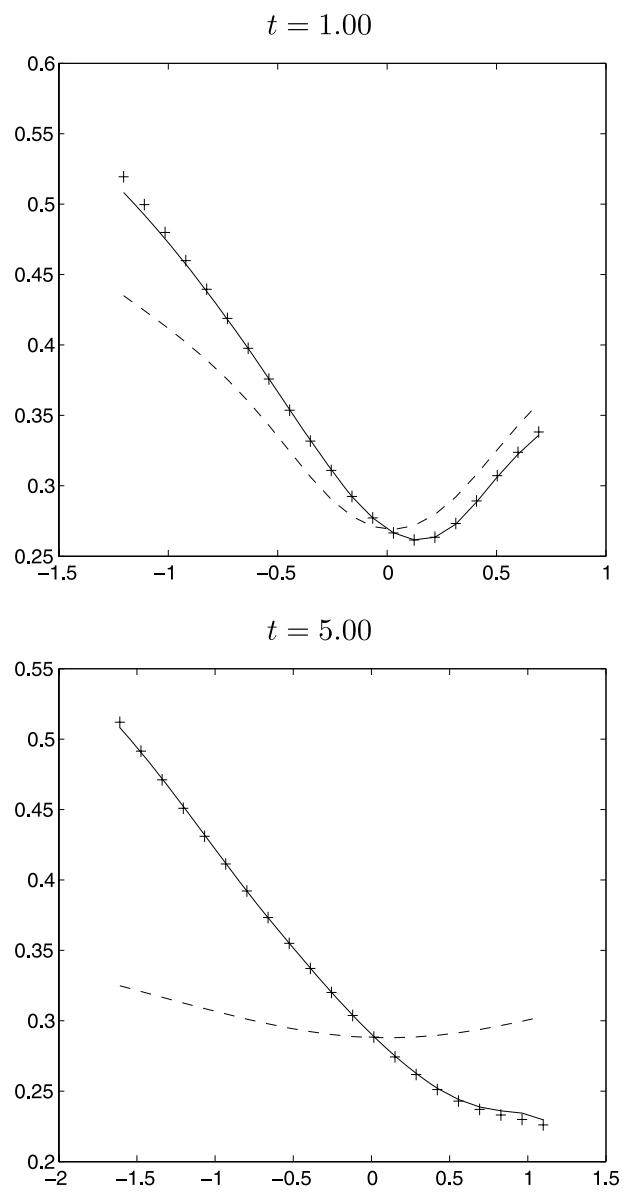

FIG. 1. Implied volatility (IV) is plotted as a function of log-strike $k:=\log K$ for the CEV-like model with Gaussian-type jumps of Section 6.1.1. The solid lines corresponds to the IV induced by $u^{(3)}(t, x)$, which is computed using the one-point Taylor expansion (see Example 1). The dashed lines corresponds to the IV induced by $u^{(0)}(t, x)$ (again, using the usual one-point Taylor series expansion). The crosses correspond to the $I V$ induced by $u^{(\mathrm{MC})}(t, x)$, which is the price obtained from the Monte Carlo simulation.

Comparing one-point Taylor and Hermite expansions. As choosing different basis functions results in distinct option-pricing approximations, one might wonder: which choice of basis functions provides the most accurate approximation of option prices and implied volatilities? We investigate this question in Figure 2. In the left column, using the parameters in (35), we plot $\operatorname{IV}\left[u^{(n)}(t, x ; K)\right], t=0.5$, $n=\{0,1,2,3,4\}$ where $u^{(n)}(t, x ; K)$ is computed using both the one-point Taylor series basis functions (Example 1) and the Hermite polynomial basis functions (Example 3$)$. We also plot $\operatorname{IV}\left[u^{(\mathrm{MC})}(t, x ; K)\right]$, the implied volatility obtained by Monte Carlo simulation. For comparison, in the right column, we plot the function 
TABLE 1

Prices $(u)$ and Implied volatility $(\operatorname{IV}[u])$ as a function of time to maturity $t$ and log-strike $k:=\log K$ for the CEV-like model with Gaussian-type jumps of Section 6.1.1. The approximate price $u^{(3)}$ is computed using the (usual) one-point Taylor expansion (see Example 1). For comparison, we provide the $95 \%$ confidence intervals for prices and implied volatilities, which we obtain from the Monte Carlo simulation

\begin{tabular}{lrcccc}
\hline $\boldsymbol{t}$ & $\boldsymbol{k}$ & $\boldsymbol{u}^{(\mathbf{3})}$ & $\boldsymbol{u}$ MC-95\% c.i. & IV $\left[\boldsymbol{u}^{(\mathbf{3})}\right]$ & IV MC-95\% c.i. \\
\hline 0.2500 & -0.6931 & 0.0006 & $0.0006-0.0007$ & 0.5864 & $0.5856-0.5901$ \\
& -0.4185 & 0.0024 & $0.0024-0.0025$ & 0.4563 & $0.4553-0.4583$ \\
& -0.1438 & 0.0111 & $0.0110-0.0112$ & 0.2875 & $0.2865-0.2883$ \\
& 0.1308 & 0.1511 & $0.1508-0.1513$ & 0.2595 & $0.2573-0.2608$ \\
1.0000 & 0.4055 & 0.5028 & $0.5024-0.5030$ & 0.4238 & $0.4152-0.4288$ \\
& -1.2040 & 0.0009 & $0.0009-0.0010$ & 0.5115 & $0.5176-0.5210$ \\
& -0.7297 & 0.0046 & $0.0047-0.0048$ & 0.4174 & $0.4178-0.4199$ \\
& -0.2554 & 0.0314 & $0.0313-0.0316$ & 0.3109 & $0.3102-0.3117$ \\
3.0000 & 0.2189 & 0.2781 & $0.2775-0.2784$ & 0.2638 & $0.2620-0.2649$ \\
& 0.6931 & 1.0034 & $1.0030-1.0041$ & 0.3358 & $0.3296-0.3459$ \\
& -1.3863 & 0.0074 & $0.0081-0.0083$ & 0.4758 & $0.4851-0.4870$ \\
& -0.8664 & 0.0224 & $0.0224-0.0227$ & 0.4031 & $0.4029-0.4045$ \\
& -0.3466 & 0.0776 & $0.0773-0.0779$ & 0.3280 & $0.3274-0.3288$ \\
5.0000 & 0.1733 & 0.3097 & $0.3094-0.3107$ & 0.2690 & $0.2685-0.2703$ \\
& 0.6931 & 1.0155 & $1.0150-1.0169$ & 0.2558 & $0.2540-0.2604$ \\
& -1.6094 & 0.0160 & $0.0164-0.0166$ & 0.5082 & $0.5111-0.5128$ \\
& -0.9324 & 0.0439 & $0.0436-0.0440$ & 0.4118 & $0.4107-0.4121$ \\
& -0.2554 & 0.1504 & $0.1497-0.1507$ & 0.3203 & $0.3194-0.3208$ \\
& 0.4216 & 0.6139 & $0.6123-0.6142$ & 0.2521 & $0.2500-0.2524$ \\
& 1.0986 & 2.0050 & $2.0032-2.0057$ & 0.2297 & $0.2163-0.2342$ \\
\hline
\end{tabular}

$f$ as well as $f_{\text {Taylor }}^{(n)}$ and $f_{\text {Hermite }}^{(n)}$ where

(36)

$$
f(x)=e^{2(\beta-1) x}, \quad f_{\text {Taylor }}^{(n)}(x):=\sum_{m=0}^{n} \frac{1}{m !} \partial^{m} f(\bar{x})(x-\bar{x})^{m},
$$

$$
f_{\text {Hermite }}^{(n)}(x):=\sum_{m=0}^{n} \frac{1}{m !}\left\langle H_{m}, f\right\rangle H_{m}(x) .
$$

From Figure 2, we observe that, for every $n$, the Taylor series expansion $f_{\text {Taylor }}^{(n)}$ provides a better approximation of the function $f$ (at least locally) than does the Hermite polynomial expansion $f_{\text {Hermite }}^{(n)}$ In turn, the implied volatilities resulting from the Taylor series basis functions $\operatorname{IV}\left[u^{(n)}(t, x ; K)\right]$ more accurately approximate $\operatorname{IV}\left[u^{(\mathrm{MC})}(t, x ; K)\right]$ than do the implied volatilities resulting from the Hermite basis functions. The implied volatilities resulting from the two-point Taylor series price approximation (not shown in the figure for clarity), are nearly indistin- 


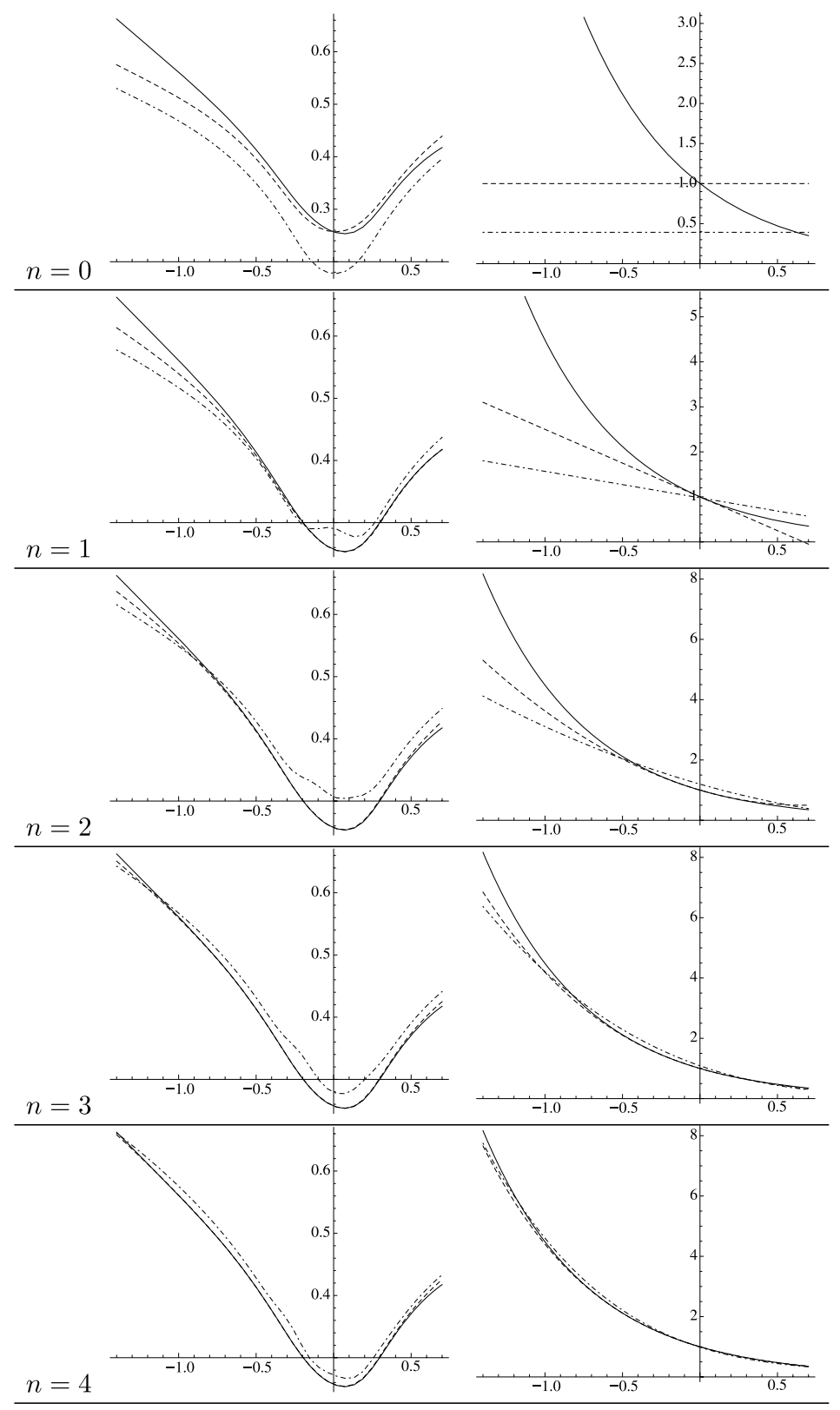

FIG. 2. Left: for the model considered in Section 6.1 .1 and for a fixed maturity $t=0.5$, implied volatility is plotted as a function of log-strike. The dashed line corresponds to $\operatorname{IV}\left[u^{(n)}\right]$ where $u^{(n)}$ is computed using Taylor series basis functions (Example 1). The dot-dashed line corresponds to $\mathrm{IV}\left[u^{(n)}\right]$ where $u^{(n)}$ is computed using Hermite polynomial basis functions (Example 3$)$. The solid line corresponds to IV $\left[u^{(\mathrm{MC})}\right]$. Right: $f(x)=e^{2(\beta-1) x}$ (solid) and its $n$th order Taylor series and Hermite polynomial approximations $f_{\text {Taylor }}^{(n)}(x)($ dotted $)$ and $f_{\text {Hermite }}^{(n)}(x)$ (dot-dashed); see equation (36). 
guishable implied volatilities induced by the (usual) one-point Taylor series price approximation.

Computational speed, accuracy and robustness. In order for a method of obtaining approximate option prices to be useful to practitioners, the method must be fast, accurate and work over a wide range of model parameters. In order to test the speed, accuracy and robustness of our method, we select model parameters at random from uniform distributions within the following ranges:

$$
\begin{aligned}
& \delta \in[0.0,0.6], \quad \beta \in[0.0,1.0], \quad \lambda \in[0.0,1.0], \quad m \in[-1.0,0.0], \\
& \eta \in[0.0,1.0] .
\end{aligned}
$$

Using the obtained parameters, we then compute approximate option prices $u^{(3)}$ and record computation times over a fixed range of strikes using our third-order one-point Taylor expansion (Example 1). As the exact price of a call option is not available, we also compute option prices by Monte Carlo simulation. The results are displayed in Tables 2 and 3. The tables show that our third order price approximation $u^{(3)}$ consistently falls within the $95 \%$ confidence interval obtained from the Monte Carlo simulation. Moreover, using a $2.4 \mathrm{GHz}$ laptop computer, an approximate call price $u^{(3)}$ can be computed in only $\approx 0.05$ seconds. This is only four to five times larger than the amount of time it takes to compute a similar option price using standard Fourier methods in an exponential Lévy setting.

6.1.2. Variance-Gamma Lévy measure. In our second numerical experiment, we consider the case of Variance-Gamma jumps. That is, $\mathcal{N}(d z)$ given by (34). We fix the following parameters:

$$
\begin{aligned}
\delta & =0.0, \quad \beta=0.25, \quad \theta=-0.3, \quad \rho=0.3, \quad \kappa=0.15, \\
S_{0} & =e^{x}=1 .
\end{aligned}
$$

Note that, by letting $\delta=0$, we have set the diffusion component of $X$ to zero: $a(x)=0$. Thus, $X$ is a pure-jump Lévy-type process. Using Corollary 1 , we compute the approximate prices $u^{(0)}(t, x ; K)$ and $u^{(2)}(t, x ; K)$ of a series of European puts over a range of strikes and with maturities $t \in\{0.5,1.0\}$. To compute $u^{(i)}$, $i \in\{0,2\}$, we use the two-point Taylor series expansion (Example 2). We also compute the put prices by Monte Carlo simulation. For the Monte Carlo simulation, we use a time-step of $10^{-3}$ years and we simulate $10^{6}$ sample paths. At each time-step, we update $X$ using the following algorithm:

$$
\begin{aligned}
X_{t+\Delta t} & =X_{t}+b\left(X_{t}\right) \Delta t+\gamma^{+}\left(X_{t}\right)-\gamma^{-}\left(X_{t}\right), \quad I(x)=e^{2(\beta-1) x}, \\
b(x) & =-\frac{I(x)}{\kappa}\left(\log \left(\frac{\lambda_{-}}{1+\lambda_{-}}\right)+\log \left(\frac{\lambda_{+}}{\lambda_{+}-1}\right)\right), \\
\gamma^{ \pm}(x) & \sim \Gamma\left(I(x) \cdot \Delta t / \kappa, 1 / \lambda_{ \pm}\right),
\end{aligned}
$$


TABLE 2

After selecting model parameters randomly, we compute call prices (u) for the CEV-like model with Gaussian-type jumps discussed in Section 6.1.1. For each strike, the approximate call price $u^{(3)}$ is computed using the (usual) one-point Taylor expansion (see Example 1) as well as by Monte Carlo simulation. The obtained prices, as well as the associated implied volatilities (IV $[u])$ are displayed above. Note that, the approximate price $u^{(3)}$ (and corresponding implied volatility) consistently falls within the $95 \%$ confidence interval obtained from the Monte Carlo simulation. We denote by $\tau^{(n)}$ the total time it takes to compute the nth order approximation of option prices $u^{(n)}$ at the five strikes displayed in the table. Because total computation time depends on processor speed, in the last column, we give the ratio $\tau^{(3)} / \tau^{(0)}$. Note that $\tau^{(0)}$ is a useful benchmark, as it corresponds to the total time it takes to compute the five call in an exponential Lévy setting (i.e., option prices with no local dependence) using standard Fourier techniques

\begin{tabular}{lrccccc}
\hline Parameters & $\boldsymbol{k}=\log \boldsymbol{K}$ & $\boldsymbol{u}^{(\mathbf{3})}$ & $\boldsymbol{u}$ MC-95\% c.i. & $\mathbf{I V}\left[\boldsymbol{u}^{(\mathbf{3})}\right]$ & $\mathbf{I V ~ M C - 9 5 \% ~ c . i . ~}$ & $\boldsymbol{\tau}^{(\mathbf{3})} / \boldsymbol{\tau}^{(\mathbf{0})}$ \\
\hline & & \multicolumn{5}{c}{$t=0.25$ years } \\
$\delta=0.5432$ & -0.6000 & 0.4552 & $0.4552-0.4553$ & 0.6849 & $0.6836-0.6869$ & 4.9787 \\
$\beta=0.3756$ & -0.3500 & 0.3123 & $0.3122-0.3124$ & 0.6230 & $0.6217-0.6242$ & \\
$\lambda=0.0518$ & -0.1000 & 0.1621 & $0.1618-0.1623$ & 0.5704 & $0.5687-0.5714$ & \\
$m=-0.5013$ & 0.1500 & 0.0496 & $0.0492-0.0500$ & 0.5240 & $0.5222-0.5266$ & \\
$\eta=0.3839$ & 0.4000 & 0.0059 & $0.0057-0.0067$ & 0.4821 & $0.4787-0.4950$ & \\
$\delta=0.1182$ & -0.6000 & 0.4566 & $0.4566-0.4567$ & 0.7257 & $0.7239-0.7271$ & 4.77419 \\
$\beta=0.9960$ & -0.3500 & 0.3137 & $0.3136-0.3139$ & 0.6391 & $0.6378-0.6405$ & \\
$\lambda=0.8938$ & -0.1000 & 0.1431 & $0.1429-0.1434$ & 0.4615 & $0.4602-0.4630$ & \\
$m=-0.4486$ & 0.1500 & 0.0032 & $0.0030-0.0037$ & 0.2013 & $0.1970-0.2073$ & \\
$\eta=0.2619$ & 0.4000 & 0.0000 & $0.0000-0.0000$ & 0.2510 & $0.2567-0.2616$ & \\
$\delta=0.3376$ & -0.6000 & 0.4621 & $0.4619-0.4621$ & 0.8462 & $0.8439-0.8478$ & 4.31915 \\
$\beta=0.4805$ & -0.3500 & 0.3190 & $0.3189-0.3192$ & 0.6949 & $0.6933-0.6968$ & \\
$\lambda=0.9610$ & -0.1000 & 0.1578 & $0.1575-0.1581$ & 0.5457 & $0.5444-0.5476$ & \\
$m=-0.2420$ & 0.1500 & 0.0451 & $0.0448-0.0456$ & 0.4990 & $0.4974-0.5021$ & \\
$\eta=0.5391$ & 0.4000 & 0.0155 & $0.0152-0.0162$ & 0.6006 & $0.5981-0.6080$ & \\
$\delta=0.2469$ & -0.6000 & 0.4592 & $0.4591-0.4593$ & 0.7871 & $0.7857-0.7900$ & 4.46032 \\
$\beta=0.1875$ & -0.3500 & 0.3100 & $0.3099-0.3102$ & 0.5965 & $0.5950-0.5986$ & \\
$\lambda=0.4229$ & -0.1000 & 0.1341 & $0.1338-0.1343$ & 0.4083 & $0.4069-0.4096$ & \\
$m=-0.2823$ & 0.1500 & 0.0306 & $0.0302-0.0309$ & 0.4149 & $0.4126-0.4168$ & \\
$\eta=0.7564$ & 0.4000 & 0.0176 & $0.0171-0.0179$ & 0.6213 & $0.6171-0.6244$ & \\
\hline
\end{tabular}

where $\Gamma(a, b)$ is a Gamma-distributed random variable with shape parameter $a$ and scale parameter $b$. Note that this is equivalent to considering a VG-type process with state-dependent parameters

$$
\kappa^{\prime}(x):=\kappa / I(x), \quad \theta^{\prime}(x):=\theta I(x), \quad \rho^{\prime}(x):=\rho \sqrt{I(x)} .
$$

These state-dependent parameters result in state-independent $\lambda_{ \pm}$(i.e., $\lambda_{ \pm}$remain constant). Once again, since implied volatilities rather than prices are the quantity of primary interest, we convert prices to implied volatilities by inverting the Black-Scholes formula numerically. The results are plotted in Figure 3. We ob- 
TABLE 3

After selecting model parameters randomly, we compute call prices $(u)$ for the CEV-like model with Gaussian-type jumps discussed in Section 6.1.1. For each strike, the approximate call price $u^{(3)}$ is computed using the (usual) one-point Taylor expansion (see Example 1) as well as by Monte Carlo simulation. The obtained prices, as well as the associated implied volatilities $(\operatorname{IV}[u])$ are displayed above. Note that the approximate price $u^{(3)}$ (and corresponding implied volatility) consistently falls within the $95 \%$ confidence interval obtained from the Monte Carlo simulation. We denote by $\tau^{(n)}$ the total time it takes to compute the nth order approximation of option prices $u^{(n)}$ at the five strikes displayed in the table. Because total computation time depends on processor speed, in the last column, we give the ratio $\tau^{(3)} / \tau^{(0)}$. Note that $\tau^{(0)}$ is a useful benchmark, as it corresponds to the total time it takes to compute the five call in an exponential Lévy setting (i.e., option prices with no local dependence) using standard Fourier techniques

\begin{tabular}{lrccccc}
\hline Parameters & $\boldsymbol{k}=\log \boldsymbol{K}$ & $\boldsymbol{u}^{(\mathbf{3})}$ & $\boldsymbol{u}$ MC-95\% c.i. & $\mathbf{I V}\left[\boldsymbol{u}^{(\mathbf{3})}\right]$ & IV MC-95\% c.i. & $\boldsymbol{\tau}^{(\mathbf{3})} / \boldsymbol{\tau}^{(\mathbf{0})}$ \\
\hline & & \multicolumn{5}{c}{$t=1.00$ years } \\
$\delta=0.5806$ & -1.0000 & 0.6487 & $0.6486-0.6488$ & 0.7306 & $0.7294-0.7319$ & 4.97872 \\
$\beta=0.5829$ & -0.6000 & 0.5001 & $0.5000-0.5004$ & 0.6719 & $0.6711-0.6734$ & \\
$\lambda=0.0367$ & -0.2000 & 0.3220 & $0.3216-0.3224$ & 0.6167 & $0.6157-0.6182$ & \\
$m=-0.6622$ & 0.2000 & 0.1512 & $0.1507-0.1520$ & 0.5649 & $0.5636-0.5671$ & \\
$\eta=0.2984$ & 0.6000 & 0.0413 & $0.0408-0.0428$ & 0.5166 & $0.5145-0.5219$ & \\
$\delta=0.3921$ & -1.0000 & 0.6556 & $0.6555-0.6561$ & 0.8022 & $0.8014-0.8075$ & 4.54839 \\
$\beta=0.1271$ & -0.6000 & 0.5012 & $0.5011-0.5018$ & 0.6779 & $0.6772-0.6809$ & \\
$\lambda=0.4176$ & -0.2000 & 0.3052 & $0.3051-0.3060$ & 0.5655 & $0.5651-0.5678$ & \\
$m=-0.1661$ & 0.2000 & 0.1188 & $0.1184-0.1198$ & 0.4832 & $0.4822-0.4858$ & \\
$\eta=0.5823$ & 0.6000 & 0.0299 & $0.0296-0.0315$ & 0.4708 & $0.4694-0.4772$ & \\
$\delta=0.5803$ & -1.0000 & 0.6679 & $0.6677-0.6681$ & 0.9122 & $0.9108-0.9140$ & 4.3125 \\
$\beta=0.2426$ & -0.6000 & 0.5237 & $0.5236-0.5243$ & 0.7916 & $0.7913-0.7943$ & \\
$\lambda=0.5926$ & -0.2000 & 0.3436 & $0.3431-0.3441$ & 0.6830 & $0.6814-0.6845$ & \\
$m=-0.0877$ & 0.2000 & 0.1592 & $0.1581-0.1596$ & 0.5851 & $0.5823-0.5862$ & \\
$\eta=0.3236$ & 0.6000 & 0.0373 & $0.0358-0.0379$ & 0.5009 & $0.4949-0.5033$ & \\
$\delta=0.3096$ & -1.0000 & 0.6323 & $0.6323-0.6324$ & 0.36740 & $0.3680-0.3708$ & 4.9257 \\
$\beta=0.6417$ & -0.6000 & 0.4554 & $0.4553-0.4554$ & 0.34493 & $0.3442-0.3456$ & \\
$\lambda=0.3806$ & -0.2000 & 0.2283 & $0.2281-0.2284$ & 0.32159 & $0.3208-0.3221$ & \\
$m=-0.02824$ & 0.2000 & 0.0495 & $0.0491-0.0500$ & 0.29930 & $0.2980-0.3006$ & \\
$\eta=0.0122$ & 0.6000 & 0.0021 & $0.0015-0.0027$ & 0.27807 & $0.2655-0.2888$ & \\
\hline
\end{tabular}

serve that $\operatorname{IV}\left[u^{(2)}(t, x ; K)\right]$ agrees almost exactly with $\operatorname{IV}\left[u^{(\mathrm{MC})}(t, x ; K)\right]$. Values for $u^{(2)}(t, x ; K)$, the associated implied volatilities $\operatorname{IV}\left[u^{(2)}(t, x ; K)\right]$ and the $95 \%$ confidence intervals resulting from the Monte Carlo simulation can be found in Table 4.

7. Conclusion. In this paper, we consider an asset whose risk-neutral dynamics are described by an exponential Lévy-type martingale subject to default. This class includes nearly all nonnegative Markov processes. In this very general setting, we provide a family of approximations - one for each choice of the basis 

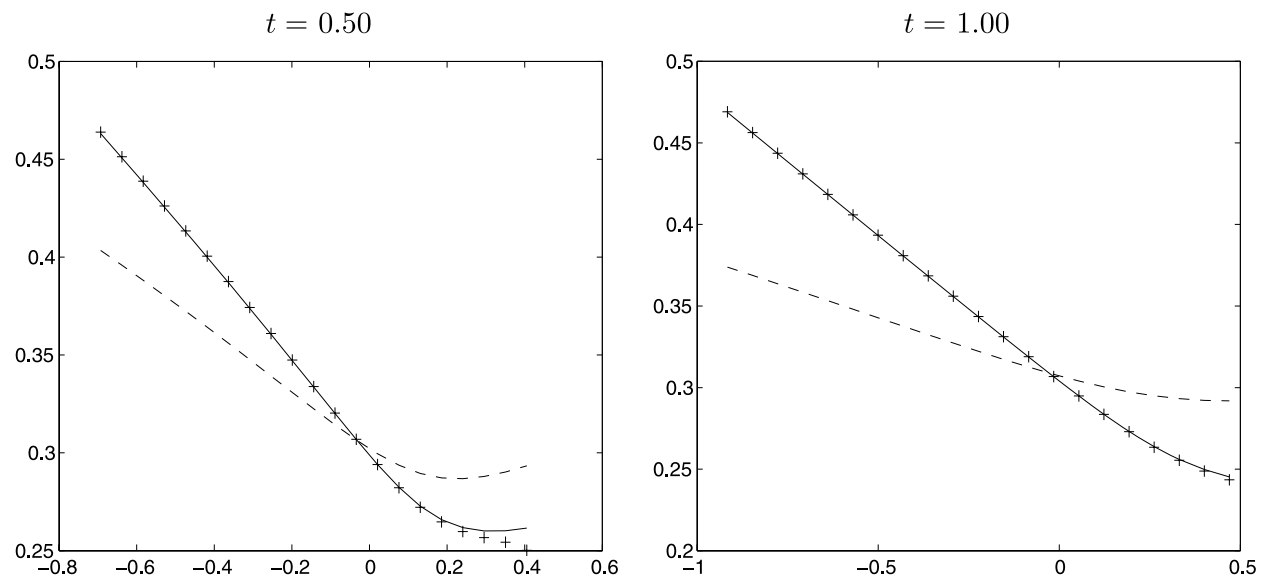

FIG. 3. Implied volatility (IV) is plotted as a function of log-strike $k:=\log K$ for the CEV-like model with Variance-Gamma-type jumps of Section 6.1.2. The solid lines corresponds to the IV induced by $u^{(2)}(t, x)$, which is computed using the two-point Taylor expansion (see Example 2). The dashed lines corresponds to the IV induced by $u^{(0)}(t, x)$ (again, computed using the two-point Taylor series expansion). The crosses correspond to the $I V$ induced by $u^{(\mathrm{MC})}(t, x)$, which is the price obtained from the Monte Carlo simulation.

functions (i.e., Taylor, two-point Taylor, $L^{2}$ basis, etc.) - for (i) the transition density of the underlying (ii) European-style option prices and their sensitivities and (iii) defaultable bond prices and their credit spreads. For the transition densities, and thus for option and bond prices as well, we establish the accuracy of our asymptotic expansion.

TABLE 4

Prices $(u)$, Implied volatilities (IV $[u])$ and the corresponding confidence intervals from Figure 3

\begin{tabular}{lccccc}
\hline $\boldsymbol{t}$ & $\boldsymbol{k}$ & $\boldsymbol{u}^{(\mathbf{2})}$ & $\boldsymbol{u}$ MC-95\% c.i. & IV $\left[\boldsymbol{u}^{(\mathbf{2})}\right]$ & IV MC-95\% c.i. \\
\hline 0.5000 & -0.6931 & 0.0014 & $0.0014-0.0015$ & 0.4631 & $0.4624-0.4652$ \\
& -0.4185 & 0.0070 & $0.0070-0.0071$ & 0.4000 & $0.3995-0.4014$ \\
& -0.1438 & 0.0363 & $0.0362-0.0365$ & 0.3336 & $0.3331-0.3346$ \\
& 0.1308 & 0.1702 & $0.1697-0.1704$ & 0.2727 & $0.2707-0.2736$ \\
1.0000 & 0.4055 & 0.5011 & $0.5004-0.5012$ & 0.2615 & $0.2291-0.2646$ \\
& -0.9163 & 0.0028 & $0.0027-0.0028$ & 0.4687 & $0.4678-0.4702$ \\
& -0.5697 & 0.0109 & $0.0109-0.0110$ & 0.4057 & $0.4050-0.4068$ \\
& -0.2231 & 0.0473 & $0.0472-0.0476$ & 0.3434 & $0.3428-0.3444$ \\
& 0.1234 & 0.1970 & $0.1965-0.1974$ & 0.2836 & $0.2825-0.2847$ \\
& 0.4700 & 0.6033 & $0.6025-0.6037$ & 0.2452 & $0.2355-0.2506$ \\
\hline
\end{tabular}




\section{APPENDIX A: PROOF OF THEOREM 1}

By hypothesis $v_{n} \in L^{1}(\mathbb{R}, d x)$, and thus, by standard Fourier transform properties we the following relation holds:

$$
\mathcal{F}\left(\mathcal{A}_{k} v_{n}(t, \cdot)\right)(\xi)=\phi_{k}(t, \xi) \widehat{v}_{n}(t, \xi), \quad n, k \geq 0 .
$$

We now Fourier transform equation (24). At the left-hand side, we have

$$
\mathcal{F}\left(\left(\partial_{t}+\mathcal{A}_{0}\right) v_{n}(t, \cdot)\right)(\xi)=\left(\partial_{t}+\phi_{0}(t, \xi)\right) \widehat{v}_{n}(t, \xi) .
$$

Next, for the right-hand side of (24), we get

$$
\begin{aligned}
-\sum_{k=1}^{n} & \int_{\mathbb{R}} d x\left(\frac{e^{-i \xi x}}{\sqrt{2 \pi}} B_{k}(x)\right) \mathcal{A}_{k} v_{n-k}(t, x) \\
= & -\sum_{k=1}^{n} \int_{\mathbb{R}} d x\left(B_{k}\left(i \partial_{\xi}\right) \frac{e^{-i \xi x}}{\sqrt{2 \pi}}\right) \mathcal{A}_{k} v_{n-k}(t, x) \\
& =-\sum_{k=1}^{n} B_{k}\left(i \partial_{\xi}\right) \mathcal{F}\left(\mathcal{A}_{k} v_{n-k}(t, \cdot)\right)(\xi)
\end{aligned}
$$

[by (37)]

$$
=-\sum_{k=1}^{n} B_{k}\left(i \partial_{\xi}\right)\left(\phi_{k}(t, \xi) \widehat{v}_{n-k}(t, \xi)\right) .
$$

Thus, we have the following ODEs (in $t$ ) for $\widehat{v}_{n}(t, \xi)$ :

$$
\begin{aligned}
& \left(\partial_{t}+\phi_{0}(t, \xi)\right) \widehat{v}_{0}(t, \xi)=0, \quad \widehat{v}_{0}(T, \xi)=\widehat{h}(\xi), \\
& \left(\partial_{t}+\phi_{0}(t, \xi)\right) \widehat{v}_{n}(t, \xi)=-\sum_{k=1}^{n} B_{k}\left(i \partial_{\xi}\right)\left(\phi_{k}(t, \xi) \widehat{v}_{n-k}(t, \xi)\right),
\end{aligned}
$$

$$
\widehat{v}_{n}(T, \xi)=0 .
$$

One can easily verify (e.g., by substitution) that the solutions of (38) and (39) are given by (25) and (26), respectively.

\section{APPENDIX B: MATHEMATICA CODE}

The following Mathematica code can be used to generate the $\widehat{u}_{n}(t, \xi)$ automatically for Taylor series basis functions: $B_{n}(x)=\left(x-x_{0}\right)^{n}$. We have

$$
\begin{aligned}
& \mathrm{B}\left[\mathrm{n}_{-}, \mathrm{x}_{-}, \mathrm{x} 0_{-}\right]=(\mathrm{x}-\mathrm{x} 0)^{\wedge} \mathrm{n} ; \\
& \mathrm{Bop}\left[\mathrm{n}_{-}, \xi_{-}, \mathrm{x} 0_{-}, \mathrm{ff} \mathrm{f}_{-}\right] \\
& \quad:=\text { Module }[\text { mat }, \operatorname{dim}, \mathrm{x}\},
\end{aligned}
$$




$$
\begin{aligned}
& \text { mat }=\text { CoefficientList }[\mathrm{B}[n, x, \mathrm{x} 0], \mathrm{x}] \text {; } \\
& \operatorname{dim}=\text { Dimensions[mat }] \text {; } \\
& \left.\sum_{m=1}^{\operatorname{dim}[[1]]} \operatorname{mat}[[m]](i)^{\wedge}(m-1) D[f f,\{\xi, m-1\}]\right] \text {; } \\
& \mathrm{u}\left[\mathrm{n}_{-}, \mathrm{t}_{-}, \xi_{-}, \mathrm{x} \mathrm{O}_{-}, \mathrm{k}_{-}\right] \\
& :=\operatorname{Exp}[\mathrm{t} \phi[0, \xi, \mathrm{x} 0]] \\
& \times \sum_{\mathrm{m}=1}^{\mathrm{n}} \int_{0}^{\mathrm{t}} \operatorname{Exp}[-\mathrm{s} \phi[0, \xi, \mathrm{x} 0]] \\
& \times(\mathrm{Bop}[\mathrm{m}, \xi, \mathrm{x} 0, \phi[\mathrm{m}, \xi, \mathrm{x} 0] \mathrm{u}[\mathrm{n}-\mathrm{m}, \mathrm{s}, \xi, \mathrm{x} 0, \mathrm{k}]]) \mathrm{ds} ; \\
& \mathrm{u}\left[0, \mathrm{t}_{-}, \xi_{-} \mathrm{x} 0_{-}, \mathrm{k}_{-}\right]=\operatorname{Exp}[\mathrm{t} \phi[0, \xi, \mathrm{x} 0]] \mathrm{h}[\xi, \mathrm{k}] .
\end{aligned}
$$

The function $\widehat{u}_{n}(t, \xi)$ is now computed explicitly by typing $\mathrm{u}\left[\mathrm{n}_{-}, \mathrm{t}_{-}, \xi_{-}, \mathrm{x} 0_{-}\right.$, $\mathrm{k}_{-}$] and pressing Shift + Enter. Note that the function $\widehat{u}_{n}(t, \xi)$ can depend on a parameter $k$ (e.g., log-strike) through the Fourier transform of the payoff function $\widehat{h}(\xi, k)$. To compute $\widehat{u}_{n}(t, \xi)$ using other basis functions, one simply has to replace the first line in the code. For example, for Hermite polynomial basis functions, one rewrites the top line as

$$
\mathrm{B}\left[\mathrm{n}_{-}, \mathrm{x}_{-}, \mathrm{x}_{-}\right]=\frac{1}{\sqrt{(2 \mathrm{n}) ! ! \sqrt{\pi}}} \operatorname{HermiteH}[\mathrm{n}, \mathrm{x}-\mathrm{x} 0] \text {; }
$$

where HermiteH[n, $\mathrm{x}]$ is the Mathematica command for the $n$th Hermite polynomial $H_{n}(x)$ [note that Mathematica does not normalize the Hermite polynomials as we do in equation (21)].

\section{APPENDIX C: SKETCH OF THE PROOF OF THEOREM 2}

PROOF. For sake of simplicity, we only provide a sketch of the proof for the case $\delta(t, x) \equiv \delta, \mu(t, x) \equiv \mu$ and $N=1$. For the complete and detailed proof, we refer to the companion paper Lorig, Pagliarani and Pascucci (2013).

The main idea of the proof is to use our expansion as a parametrix. That is, our expansion will be the starting point of the classical iterative method introduced by Levi (1907) to construct the fundamental solution $p(t, x ; T, y)$. Specifically, as in Pagliarani, Pascucci and Riga (2013), we take as a parametrix our $N$ th order approximation $p^{(N)}(t, x ; T, y)$ with basis functions $B_{n}=(x-\bar{x})^{n}$ and with $\bar{x}=y$. By analogy with the classical approach [see, e.g., Friedman (1964) and Di Francesco and Pascucci (2005), Pascucci (2011) for the purely diffusive case, or Garroni and Menaldi (1992) for the integro-differential case], we have

$$
p(t, x ; T, y)=p^{(1)}(t, x ; T, y)+\int_{t}^{T} \int_{\mathbb{R}} p^{(0)}(t, x ; s, \xi) \Phi(s, \xi ; T, y) d \xi d s,
$$


where $\Phi$ is determined by imposing the condition

$$
\begin{aligned}
0= & L p(t, x ; T, y) \\
= & L p^{(1)}(t, x ; T, y) \\
& +\int_{t}^{T} \int_{\mathbb{R}} L p^{(0)}(t, x ; s, \xi) \Phi(s, \xi ; T, y) d \xi d s-\Phi(t, x ; T, y) .
\end{aligned}
$$

Equivalently, we have

$$
\Phi(t, x ; T, y)=L p^{(1)}(t, x ; T, y)+\int_{t}^{T} \int_{\mathbb{R}} L p^{(0)}(t, x ; s, \xi) \Phi(s, \xi ; T, y) d \xi d s,
$$

and, therefore, by iteration

$$
\Phi(t, x ; T, y)=\sum_{n=0}^{\infty} Z_{n}(t, x ; T, y),
$$

where

$$
\begin{aligned}
Z_{0}(t, x ; T, y) & :=L p^{(1)}(t, x ; T, y) \\
Z_{n+1}(t, x ; T, y) & :=\int_{t}^{T} \int_{\mathbb{R}} L p^{(0)}(t, x ; s, \xi) Z_{n}(s, \xi ; T, y) d \xi d s .
\end{aligned}
$$

The proof of Theorem 2 then is based on some pointwise bounds for each term $Z_{n}$ in (41). These bounds, summarized in the next two propositions, can be combined with formula (40) to obtain the estimate for $\left|p(t, x ; T, y)-p^{(1)}(t, x ; T, y)\right|$.

PROPOSITION 2. There exists a positive constant $C$, only dependent on $\tau, m$ and $M$, such that

$$
p^{(0)}(t, x ; T, y) \leq C \bar{\Gamma}(t, x ; T, y), \quad 0 \leq t<T \leq \tau,
$$

for any $x, y \in \mathbb{R}$ and $t, T \in \mathbb{R}$ with $0 \leq t<T \leq \tau$.

Proposition 3. There exists a positive constant $C$, only dependent on $\tau, m, M$ and $\left(\left\|\lambda_{i}\right\|_{\infty},\left\|\gamma_{i}\right\|_{\infty},\left\|a_{i}\right\|_{\infty}\right)_{i=1,2}$, such that

$$
\left|Z_{n}(t, x ; T, y)\right| \leq \frac{C^{n+1}(T-t)^{n / 2}}{\sqrt{n !}}\left(1+\left\|\lambda_{1}\right\|_{\infty} \mathrm{e}^{n+1}\right) \bar{\Gamma}(t, x ; T, y),
$$

for any $n \geq 0, x, y \in \mathbb{R}$ and $t, T \in \mathbb{R}$ with $0 \leq t<T \leq \tau$.

The proofs of Propositions 2 and 3 are rather technical and are based on several global pointwise estimates for the fundamental solution of a constant coefficient integro-differential operator of the form (30), along with the semigroup property

$$
\int_{\mathbb{R}} \mathrm{e}^{k} \bar{\Gamma}(t, x ; s, \xi) \mathrm{C}^{N} \bar{\Gamma}(s, \xi ; T, y) d \xi=\mathrm{e}^{k+N} \bar{\Gamma}(t, x ; T, y), \quad k, N \geq 0
$$


We refer to Lorig, Pagliarani and Pascucci (2013) for detailed proofs.

Now, by equations (40), (41) and Proposition 3, we have

$$
\begin{aligned}
&\left|p(t, x ; T, y)-p^{(1)}(t, x ; T, y)\right| \\
& \leq \sum_{n=0}^{\infty} \frac{C^{n+1}}{\sqrt{n !}} \int_{t}^{T}(T-s)^{n / 2} \int_{\mathbb{R}} p^{(0)}(t, x ; s, \xi) \\
& \quad \times\left(1+\left\|\lambda_{1}\right\|_{\infty} \mathrm{C}^{n+1}\right) \bar{\Gamma}(s, \xi ; T, y) d \xi d s
\end{aligned}
$$

(by Proposition 2)

$$
\leq \sum_{n=0}^{\infty} \frac{C^{n+1}}{\sqrt{n !}} \int_{t}^{T}(T-s)^{n / 2} \int_{\mathbb{R}} \bar{\Gamma}(t, x ; s, \xi)\left(1+\left\|\lambda_{1}\right\|_{\infty} \mathrm{e}^{n+1}\right) \bar{\Gamma}(s, \xi ; T, y) d \xi d s
$$

[by the semigroup property (42)]

$$
=2(T-t)\left(\sum_{n=0}^{\infty} \frac{C^{n+1}(T-t)^{n / 2}}{\sqrt{n !}}\left(1+\left\|\lambda_{1}\right\|_{\infty} \mathrm{e}^{n+1}\right) \bar{\Gamma}(t, x ; T, y)\right),
$$

for any $x, y \in \mathbb{R}$ and $t, T \in \mathbb{R}$ with $0 \leq t<T \leq \tau$. Since

$$
\sum_{n=0}^{\infty} \frac{C^{n+1}(T-t)^{n / 2}}{\sqrt{n !}} \mathrm{e}^{n+1} \bar{\Gamma}(t, x ; T, y),
$$

can be easily checked to be convergent, this completes the proof.

Acknowledgment. The authors would like to extend their thanks to an anonymous referee, whose comments and suggestions helped to improve this paper.

\section{REFERENCES}

Andersen, L. and Andreasen, J. (2000). Jump-diffusion processes: Volatility smile fitting and numerical methods for option pricing. Review of Derivatives Research 4 231-262.

Benhamou, E., Gobet, E. and Miri, M. (2009). Smart expansion and fast calibration for jump diffusions. Finance Stoch. 13 563-589. MR2519844

Bielecki, T. R. and RutKowski, M. (2002). Credit Risk: Modelling, Valuation and Hedging. Springer, Berlin. MR1869476

BoyARChEnKo, S. I. and LEVEndorskil̆, S. Z. (2002). Non-Gaussian Merton-Black-Scholes Theory. Advanced Series on Statistical Science \& Applied Probability 9. World Scientific, River Edge, NJ. MR1904936

Capponi, A., Pagliarani, S. and Vargiolu, T. (2014). Pricing vulnerable claims in a Lévy driven model. Finance Stoch. 18 755-789. MR3255750

CARR, P. and Linetsky, V. (2006). A jump to default extended CEV model: An application of Bessel processes. Finance Stoch. 10 303-330. MR2244347 
CARR, P. and MADAN, D. B. (2010). Local volatility enhanced by a jump to default. SIAM J. Financial Math. $12-15$. MR2592562

CARR, P., Geman, H., MADAN, D. and Yor, M. (2002). The fine structure of asset returns: An empirical investigation. The Journal of Business 75 305-333.

Christoffersen, P., Jacobs, K. and Ornthanalai, C. (2009). Exploring Time-Varying Jump Intensities: Evidence from S\&P500 Returns and Options. CIRANO, Montreal.

CoX, J. (1975). Notes on option pricing I: Constant elasticity of diffusions. Unpublished draft, Stanford Univ. A revised version of the paper was published by the Journal of Portfolio Management in 1996.

Di Francesco, M. and PAscuCCI, A. (2005). On a class of degenerate parabolic equations of Kolmogorov type. Appl. Math. Res. Express AMRX 3 77-116. MR2180050

DUPIRE, B. (1994). Pricing with a smile. Risk 7 18-20.

ERAKER, B. (2004). Do stock prices and volatility jump? Reconciling evidence from spot and option prices. The Journal of Finance 59 1367-1404.

ESTES, R. H. and LANCASTER, E. R. (1972). Some generalized power series inversions. SIAM J. Numer. Anal. 9 241-247. MR0303688

Friedman, A. (1964). Partial Differential Equations of Parabolic Type. Prentice-Hall, Englewood Cliffs, NJ. MR0181836

Garroni, M. G. and Menaldi, J.-L. (1992). Green Functions for Second Order Parabolic Integro-Differential Problems. Pitman Research Notes in Mathematics Series 275. Longman Scientific \& Technical, Harlow; copublished in the United States with Wiley, New York. MR1202037

Hagan, P. and Woodward, D. (1999). Equivalent Black volatilities. Appl. Math. Finance 6147 157.

HoH, W. (1998). Pseudo differential operators generating Markov processes. Habilitations-schrift, Universität Bielefeld, Bielefeld.

JACQUIER, A. and LORIG, M. (2013). The smile of certain Lévy-type models. SIAM J. Financial Math. 4 804-830. MR3121757

Jeanblanc, M., Yor, M. and Chesney, M. (2009). Mathematical Methods for Financial Markets. Springer Finance. Springer, London. MR2568861

Levi, E. E. (1907). Sulle equazioni lineari totalmente ellittiche alle derivate parziali. Rend. Circ. Mat. Palermo (2) 24 275-317.

Linetsky, V. (2006). Pricing equity derivatives subject to bankruptcy. Math. Finance 16 255-282. MR2212266

LINETSKY, V. (2007). Spectral methods in derivatives pricing, Chapter 6. In Financial Engineering (J. R. Birge and V. Linetsky, eds.). Handbooks in Operations Research and Management Science 15 223-299. Elsevier, Amsterdam.

LóPEZ, J. L. and Temme, N. M. (2002). Two-point Taylor expansions of analytic functions. Stud. Appl. Math. 109 297-311. MR1934653

Lorig, M., PAgliarani, S. and PAsCuCCI, A. (2013). Pricing approximations and error estimates for local Lévy-type models with default. Preprint. Available at arXiv:1304.1849.

MADAN, D., CARR, P. and CHANG, E. (1998). The variance gamma process and option pricing. European Finance Review 2 79-105.

Mendoza-Arriaga, R., CARr, P. and Linetsky, V. (2010). Time-changed Markov processes in unified credit-equity modeling. Math. Finance 20 527-569. MR2731407

Oksendal, B. and Sulem, A. (2005). Applied Stochastic Control of Jump Diffusions. Springer, Berlin. MR2109687

Pagliarani, S. and Pascucci, A. (2012). Analytical approximation of the transition density in a local volatility model. Cent. Eur. J. Math. 10 250-270. MR2863794

Pagliarani, S., PAscucci, A. and Riga, C. (2013). Adjoint expansions in local Lévy models. SIAM J. Financial Math. 4 265-296. MR3032943 
PAscuccI, A. (2011). PDE and Martingale Methods in Option Pricing. Bocconi \& Springer Series 2. Springer, Milan. MR2791231

M. LORIG

DEPARTMENT OF OPERATIONS RESEARCH AND FinANCIAL ENGINEERING

PRINCETON UNIVERSITY

Sherrerd Hall, RoOm 116

Princeton, New Jersey 08544

USA
S. PAgliarani

CMAP

ECOle Polytechnique

Route DE SACLAY, RoOM 5:30-04

91128 Palaiseau CEDEX

FRANCE

\author{
A. PASCUCCI \\ Department of MATHEMATICS \\ UNIVERSITY OF BOLOGNA \\ PiAZZA DI PORTA S. DONATO, 5 \\ 40126 BOLOGNA \\ ITALY \\ E-MAIL: andrea.pascucci@unibo.it
}

